\title{
Bildung als Ideal und Beredsamkeit als Praxis. Systemkollisionen um 1800
}

Die Vielfalt der Umschreibungen für das Schicksal der klassischen Rhetorik in der deutschen Spätaufklärung vermag durchaus zu faszinieren - vor allem aber bietet sich hier ein aussagekräftiger Indikator für die möglicherweise auch gewollte Unbestimmbarkeit dessen, was ars oratoria zwischen 1786 und 1848 bedeuten könnte. Eine entsprechende Analyse ihrer Theorie, Funktion und Wertung zwischen dem Ableben des frankophilen Absolutisten in Potsdam und den ersten parlamentarischen Debatten in der Paulskirche zu Frankfurt steht noch weitgehend aus. Tendenziös aber scheint sich eine Semantik des Niedergangs zu verdichten: schon ein Zeitgenosse verkündete ja 1812 ganz explizit den „Verfall“ der Beredsamkeit ${ }^{1}$ - was nuancenreich bis in die Terminologie der modernen Forschung nachklingt, wo etwa von einer fortschreitenden „Erosion“2 oder einem völligen „Aufweichen“3 die Rede ist. Gerne konstatiert man auch ganz rigoros das unwiderrufliche Ende der Rhetorik: ${ }^{4}$ Die Vorstellung eines langen Siechtums des großen antiken Erbes mit der Aussicht auf eine greifbare finale Phase übte offenbar einen starken Reiz aus, bot sich damit doch wiederholt die Gelegenheit, auf die moralische Überlegenheit des Nordens zu verweisen, der eben für die Wahrheit anstelle der Lüge eintrete bzw. für die gesunde Natürlichkeit anstelle dekadenter Künstlichkeit. Die entsprechende Betrachtung gefiel sich darin, vorwiegend auf die noble pädagogische Sorge zu verweisen, die der deutsche Biedermann um den Adressaten walten lasse, ohne sich dabei der (welschen) fintenreichen ,Überwältigung eines wehrlosen Hörers schuldig zu machen. In diesem Zusammenhang lag es nahe, das vielstrapazierte Diktum Kants aus der Kritik der Urteilskraft unter besonderer Betonung des moralischen Impetus aufzubieten und den humanistischen Topos vom ,guten Deutschen“ zu erneuern, der eben ganz von germanischer Einfachheit sei und die „hinterlistig[e]“ Kunst selbstverständlich verabscheue, „sich der

1 Müller 1967; vgl. im kritischen Überblick Hambsch 2011.

2 Von Rosen 2012. Andere Termini wie Überwindung, Abkehr oder Bruch finden sich ebenso.

3 Till 2004b: 105.

4 Peter Ptassek spricht sogar von einer umfassenden „Verdrängungsgeschichte“ der Rhetorik „Von der Antike bis zur Neuzeit“, wonach es eine ,moderne‘ Rhetorik überhaupt nie gegeben habe (Ptassek 1993). Vgl. zu einem Versuch, mit einer zeitspezifisch transformierten Rhetorik ein Epochenkriterium für die Frühe Neuzeit zu etablieren, Keller 2008.

Ә Open Access. () 2019 Andreas Keller, publiziert von De Gruyter. (cc) BY-NC-ND Dieses Werk ist lizenziert unter der Creative Commons Attribution-NonCommercial-NoDerivatives 4.0 Lizenz.

https://doi.org/10.1515/9783110676631-008 
Schwächen der Menschen zu seinen Absichten zu bedienen“. 5 Die aus solcher Warte fraglos gebotene Ersetzung einer korrupten Beredsamkeit durch die edle, in ihrer Autonomie über alle materiellen Zwecke erhabene Dichtkunst schrieb sich insbesondere die deutschnational orientierte Philologie auf die Fahnen, die dann auch nicht müde wurde, das deutsche Originalgenie als idealistische Bezugs- und Exportgröße ins Feld zu führen. Differenziertere Beobachter bevorzugten dagegen das Modell einer rhetorica latens, ${ }^{6}$ sprachen also vielmehr von einer dauerhaften Präsenz der (neutralen) Rhetorik, die aber gleichwohl unterschiedlichen historischen Transformationen unterworfen sein könne. So schlägt Dietmar Till zur Klärung des Problems sinnreich vor, eine diachrone Studie des jeweiligen Spannungsverhältnisses zwischen einer „strukturalistisch“ und einer „anthropologisch“ aufgefassten Rhetorik anzulegen. ${ }^{7}$ Tatsächlich erscheint eine solche graduelle und sorgsam an den vielfältigen Quellen orientierte Untersuchung hilfreicher als der pauschalierende Rückzug in eine begrifflich eher verwirrende Diagnostik, die um 1800 eine „rhetorica contra rhetoricam“, 8 eine „ingeniöse Anti-Rhetorik“9 oder gar eine „Entrhetorisierung der Rhetorik“10 $\mathrm{zu}$ erkennen glaubt.

\section{Wahrheit, Rhetorik und Dichtkunst}

Ein grundsätzliches Problem liegt ganz offensichtlich in der theoretischen Reduktion von Rhetorik auf die Frage einer parteigebundenen Darstellung von Wirklichkeit als einer lediglich scheinbaren Wahrheit. Die konkrete Anwendung des rhetorischen Systems im Sinne der sprachlichen Kommunikation in unterschiedlichen sozialen Anwendungsbereichen verliert dagegen an Seriosität. In der

5 Kant 1908: 327 f., Fußnote. Kant wirft der Beredsamkeit im Haupttext desselben Paragraphen auch vor, „durch den schönen Schein zu hintergehen“, „Maschinen der Überredung“ einzusetzen, „durch sinnliche Darstellung zu überschleichen“ etc.

6 Vgl. Krause 2001: 6: „Das Weiterleben der Rhetorik kann nur behauptet werden dank einem sehr weiten Begriff von Rhetorik“. Björn Hambsch verweist auf Johann Gottfried Herders im Blick auf die Homiletik unterschiedene „ganz andre Beredsamkeit“, vgl. Hambsch 2007: 101-108. Vgl. umfassend zur longue durée dieser Fragen auch Fohrmann 2004. Fohrmann bietet neben seiner generellen „Vorbemerkung“ auch ein wichtiges einleitendes Exposé (Fohrmann 2004: 323-326) zur Sektion „Writing Culture“, das mit dem anschaulichen Bild einer „Schwelle der ,Alten“ Rhetorik“ (ebd.: 324) arbeitet.

7 Vgl. Till 2004a.

8 Bornscheuer 1989: 13.

9 Ebd.: 17 (,post-rhetorische Rhetorik“).

10 Krause 2001: 301. 
Nachfolge Kants dominiert der Imperativ einer überzeitlichen oder allgemeinmenschlichen Wahrheit - etwa im Sinne der allesumfassenden Vernunft -, an der dann natürlich die Parlaments-, die Kanzel- oder Kathederrede nur unzureichend und grundsätzlich irreführend partizipieren können. In dieser Weise geht aber auch jede situativ bedingte Ingebrauchnahme der Beredsamkeit bzw. die entsprechend sprachlich gebundene Postulierung von Wahrheiten in der Politik, in der Religion oder auch in der Wissenschaft als indikatorische Größe für die Historiographie verloren. Alle wichtigen und traditionell eng mit der Rhetorik verzahnten Referenzbereiche obliegen damit der Entwertung. Auch dies beklagte bereits Adam Müller, ${ }^{11}$ der den „Verfall“ der Beredsamkeit in Deutschland mit der hier ja seit jeher kaum vorhandenen Mündlichkeit konnotierte, ganz konkret im Blick auf die politische Rede. In umgekehrter Wertung sah Immanuel Kant aber die „höchste Stufe“ der desavouierten Rhetorik immer dann erreicht, wenn der „Staat seinem Verderben zueilte und wahre patriotische Denkungsart erloschen war“, ${ }^{12}$ womit also gerade die Abwesenheit von Rhetorik als Voraussetzung für ein intaktes Staatswesen zu erachten sei. Politik als Mündlichkeit wird bereits hier, am Ursprung des Weges zur selbstverdienten Mündigkeit, folgenreich entwertet. Dies gilt nicht nur für Politik und Rhetorik - in ganz entsprechender Weise versuchten die verschiedenen Säkularisierungsdiskurse auch die Wirkungseinheit von Rhetorik und Theologie für erledigt zu erklären. Hier aber konnte jüngst Ernst Müller mit seiner eindrucksvollen Studie zu Hamann, Schleiermacher und Kierkegaard nachweisen, wie essentiell gerade zwischen 1759 und 1859 die Symbiose aus geistlichen und oratorischen Anteilen erschienen sein muss, so dass es just für diesen Zeitraum eine ganz besondere „Intensität“, ja „ein fruchtbares Nachleben“ der von der Aufklärung verworfenen Rhetorik „innerhalb der Theologie“ zu konstatieren gelte. ${ }^{13}$

Immanuel Kant zeichnet nicht nur für die Verdrängung der Rhetorik aus den genannten gesellschaftlichen Praxisbereichen, sondern wohl auch für eine weitere folgenreiche Maßnahme verantwortlich: Zur Klärung seiner Position stellt er in der Kritik der Urteilskraft die Beredsamkeit in eine Opposition mit der Dichtkunst, ${ }^{14}$ was konsequent auf die ethisch begründete Ersetzung der Ersteren durch Letztere hinausläuft. Während die Rhetorik im Sinne von Rednerkunst

11 Vor allem für die Wissenschaft sei eigentlich das Gespräch auf „gemeinschaftlichem Boden“ bzw. „Himmel“ und in „eine[r] und derselbe[n] Luft“ die grundlegende Voraussetzung (Müller 1967: 59). Vgl. auch den Beitrag von Peter Schnyder im vorliegenden Band.

12 Kant 1908: 328, Fußnote.

13 Müller 2016: 6. Denn „Gott selbst ist es, der sich verstellt, verhüllt, verbirgt, herunterlässt, akkommodiert und sich des Autors als Griffel bedient“ (ebd.: 8).

14 Vgl. Kant 1908: § 51. 
nur einen vorgeblichen Nutzen verspreche, ohne diesen dann auch tatsächlich zu gewähren, verspreche die Dichtkunst ihrerseits zunächst nichts, eröffne dem Adressaten dann aber sehr wohl ganz besonders weite Möglichkeiten einer freien Erkenntnis. Die Dichtung stelle die dafür notwendige Verbindung zwischen Sinnlichkeit und Verstand unabsichtlich her und folge dabei keinen fremden Direktiven: Sie ist autonom, während die Rhetorik äußerst engen und zumeist rein materiellen Zwecken („Lohngeschäft“) unterworfen sei und einzig auf ein kalkuliertes Ziel hinarbeite - nämlich dem schutzlosen Adressaten den Willen bzw. die subjektive,Wahrheit' des Produzenten aufzuzwingen. Dichtung hingegen gewähre Freiheit, weil sie die „Einbildungskraft in Freiheit versetzt“ ${ }^{15}$

Die solcherart etablierte ethische Hierarchie der beiden Sprachleistungen erscheint noch nicht genug, gefordert wird vielmehr ein tatkräftiger Substitutionsvorgang. Mit der von Kant und seinen Fürsprechern angestrebten Tilgung der verdammungswürdigen Rhetorik zugunsten einer entsprechend verklärten Dichtkunst ist dann jedoch - neben der Politik und der Theologie - ein dritter lebenspraktischer Traditionsbereich der Redekunst berührt: die Erziehung. Auf diesem Feld gilt es nach Kant eine vorgeblich scholastische Gelehrsamkeit, ihre zwingende mechanische Didaktik und die damit verbundene autoritäre Pädagogik zugunsten des höherwertigen Prinzips der Bildung zu überwinden. ${ }^{16}$ An diesem Konzeptbegriff partizipiert die Dichtkunst unmittelbar in Form eines zeitgenössischen Erfolgsmodells, des Bildungsromans, ${ }^{17}$ vor allem aber basiert darauf die gesamte akademische Neuorientierung in dem bislang mächtigsten deutschsprachigen Staatswesen. Das außenpolitisch stark geschwächte Preußen tritt mit seiner Hauptstadt Berlin in diesem Sinne in eine diskursive Konkurrenz zu Weimar, um nun ein von Staats wegen einzurichtendes Bildungsinstrument auf den Plan zu rufen, das Kants Forderungen umzusetzen verspricht: eine von autoritärer, also monologischer Lehre befreite Universität als ,Bildungsuniversität‘. Wilhelm von Humboldt setzt „Bildung“ mit „Wissenschaft“ gleich und fordert von der neuen Institution, sich allein von der ,reinen Idee der Wissenschaft“ leiten zu lassen. Dies habe in einem ,ungezwungene[n] und absichtslose[n] Zusammenwirken“ aller Be-

15 Ebd.: $§ 53$. Vgl. hierzu mit weiteren Hinweisen Bezzola 1993: 31.

$16 \mathrm{Ob}$ die von Ingrid Lohmann angeregte Vorstellung der vollständigen Transformation der Rhetorik zur Pädagogik zwischen 1750 und 1850 tragfähig bleibt, wäre anhand der genaueren Betrachtung der Verschiebung der Beredsamkeit in den Bereich der Bildung bzw. deren Spannungsverhältnis hinsichtlich ihrer Institutionalisierung zu diskutieren, vgl. Lohmann 1993.

17 Der Terminus geht wohl auf Karl Morgenstern (1820) zurück. 
teiligten im Sinne einer „nicht abgeschlossenen Suche“ zu erfolgen. ${ }^{18}$ Damit deutet sich die Vorstellung von einer dynamisch bzw. diskursiv zu klärenden Wahrheit an, die sowohl der negativ bewerteten, da allein der partiellen Zweckwahrheit eines einzelnen Redners dienenden, als auch der bereits vorab festgelegten und für absolut ausgegebenen dogmatischen Wahrheit entgegenstünde. Ausschließlich dieser ,Wahrheitssuche ${ }^{6}$ seien Lehrende wie Lernende verpflichtet, ${ }^{19}$ und damit einer Wahrheit, die es ohne einschränkende präskriptive Muster zu ermitteln wie zu vermitteln gälte. Konsequent erwächst hieraus der Bedarf einer entsprechend offenen Sprachpraxis, deren Verhältnis zur traditionellen Beredsamkeit zu prüfen wäre: Neue Begrifflichkeiten, Gattungstypologien und performative Diskursformen scheinen jetzt erforderlich. Damit aber lastet eine mächtige Hypothek auf der neuen Lehranstalt in der preußischen Metropole.

\section{Bildung und sprachbasierter Wissenstransfer}

Sofern es sich um ein Parallelunternehmen handeln sollte, läge der Konnex zum Bildungsroman in der ,Absichtslosigkeit' bzw. in der Vermeidung jeglichen Diktats außerindividueller Vorgaben, denn ,alle Bildung hat ihren Ursprung allein in dem Innern der Seele, und kann durch äussere Veranstaltungen nur veranlasst, nie hervorgebracht werden“, so Humboldt. ${ }^{20}$ Als das höchste Ziel der Bildung gälte demnach sowohl im Blick auf den stillen Leser in privater Umgebung als auch auf den stillen Hörer in einer staatlichen Bildungseinrichtung dessen lediglich anzuleitende Selbstfindung als Individuum. Diese aber wäre mit einer allgemeinen bzw. universalen Wahrheit zu synchronisieren, die eben nicht rhetorisch begründet zu sein braucht. Im Gegenteil könnte gerade diese universale Wahrheit durch einen autoritären Sprechakt, also eine perspektivisch zwingende Logik oder eine allzu suggestive Affektsteuerung, kurz: durch eine „äussere Veranstaltung“ verloren gehen. Die wahrheitskonforme Bildung wird demnach vollständig in die Innerlichkeit verschoben, in den autonomen Schutzraum individueller Vorstellungskraft. Das Ideal als eine übergreifende anthropologische Wahrheit kann individuell erkannt werden. Dagegen wäre jeder

18 So Humboldt in seinen Ausführungen zu den höheren wissenschaftlichen Anstalten: Humboldt 2010: 257. Vgl. zur Erläuterung der Phänomene in längerfristigen Phasen Zöllner 1989; Tschong 1991.

19 Vgl. im Kontext der Berliner Universitätsgründung 1810 die verschiedenen Beiträge in Tenorth 2012a, zur allgemeinen Orientierung Tenorth 2012b.

20 Humboldt 1851: 73. 
Ansatz $\mathrm{zu}$ einer intentionalen Formulierung von Einzelwahrheit(en) durch einen Sprecher in der Spezifik eines Diskursraumes, also ihre Bestimmung durch jeweils situative, oratorisch zu entfaltende Bedingungen in der politischen Debatte, im heilsgeschichtlichen Mahn- bzw. Trostgespräch oder eben in der pädagogischen Unterweisung, per se ausgeklammert - im Bildungsroman wie in der Bildungsanstalt. ${ }^{21}$ Sekundär bliebe somit ein quasi mechanisches Übermitteln von externem Faktenwissen, eine unterweisende Erziehung zur reinen, Nützlichkeit‘. Die Formung des Schülers als zukünftiger Akteur einer effizienten und hierarchischen Gemeinschaft obläge nicht der Bildung. Folglich stünde der gebildete Mensch sogar außerhalb der Gesellschaft: Das „Ideal der Menschheit“ erscheint nach Humboldt lediglich „in der Totalität der Individuen“,22 ganz im Sinne einer Addition von Einzelqualitäten, nicht jedoch in der gemeinschaftlich orientierten zweckgebundenen Unterordnung. Eine „normativ-allgemeinmenschliche Ausrichtung von Humboldts Bildungsideal“ fehlt, so konstatiert Jürgen Kost. Im reformierten Preußen steht jedoch das Interesse an zukünftigen Funktionsträgern aus Gründen der Staatsräson weiterhin im Vordergrund, somit bliebe eine entsprechende Autonomie der Universität von Anfang an stark eingeschränkt. ${ }^{23}$

Für Wilhelm von Humboldt bleibt Bildung ein stabilisierendes Wechselspiel zwischen innen und außen, zwischen subjektiver Selbstformung und objektiven Gegebenheiten:

\begin{abstract}
Was verlangt man von einer Nation, einem Zeitalter, von dem ganzen Menschengeschlecht, wenn man ihm seine Achtung und seine Bewunderung schenken soll? Man verlangt, dass Bildung, Weisheit und Tugend so mächtig und allgemein verbreitet, als möglich, unter ihm herrschen [...]. Beschränken sich indess auch alle diese Forderungen nur auf das innere Wesen des Menschen, so dringt doch seine Natur beständig von sich aus zu den Gegenständen ausser ihm überzugehen, und hier kommt es nun darauf an, dass er in dieser Entfremdung nicht sich selbst verliere, sondern vielmehr von allem, was er ausser sich vornimmt, immer das erhellende Licht und die wohlthätige Wärme in sein Innres zurückstrale. Zu dieser Absicht aber muss er die Masse der Gegenstände sich selbst näher bringen, diesem Stoff die Gestalt seines Geistes aufdrücken und beide einander ähnlicher machen. ${ }^{24}$
\end{abstract}

21 Mit der Ersetzung einer zweckgebundenen Beredsamkeit durch die autonome Dichtkunst erfahren die entsprechenden Sekundärbereiche der Rhetorik als anthropologisch unverzichtbare Erkenntnisräume ihre Einbindung in die Poesie: der Bildungsroman hat die diplomatischen, spirituellen und didaktischen Herausforderungen mit seinen Mitteln abzudecken, was in den Texten der Zeit ja auch entsprechend nachweisbar ist.

22 Humboldt 1980: 339f.

23 Kost 2004: 152; vgl. die entsprechenden Ausführungen ebd.: 140-152.

24 Humboldt 1968: 284. 
Wie aber sind nun diese Maximen in die Konzeption der gemeinschaftlichen Universitas einzubinden? In dem um 1800/1810 entstandenen Organon aus theoretischen Möglichkeiten (Forschung als einsame und zweckfreie Reflexion) und praktischen Erfordernissen (Lehre als zweckorientierte Ausbildung von Staatsbediensteten) stehen sich Wissen und Bildung, Kollektivität und Individualität, Universität und Universalität, aber schließlich auch Subjekt und Staat bzw. Subjekt und Nation durchaus spannungsvoll gegenüber. Das zu belehrende Subjekt unterliegt der direkten Aufsicht des Staates und ist konkret für künftige Aufgaben in dessen Dienst (Gymnasiallehre, Verwaltung, Seelsorge) vorzubereiten. Deshalb kann Rhetorik zumindest in ihrer propädeutischen Variante nicht verschwinden, vielmehr liegt es in staatlichem Interesse, normgebende Inhalte weiterhin mit erprobten präskriptiven Vortragsmustern ohne Reibungsverluste zu tradieren, ganz im Sinne der gezielten Integration des Subjekts in ein staatliches Pflichtprinzip. Der Wissenstransfer in der Lehre bliebe deshalb notwendigerweise monologisch, autoritär und dogmatisch und obläge automatisch den entsprechenden rhetorischen Techniken. Der idealistische Anspruch der Universität, dem Freiheitsgedanken Kants verpflichtet, kann bezeichnenderweise trotz der Kant'schen Vorbehalte die Rhetorik nicht gänzlich ausschalten. Anstelle der wertenden Diskurse über ihre Wirkung und Gefahr gilt es deshalb besser den entsprechend differenzierten Grad ihres verbleibenden praktischen Einsatzes zu verfolgen. Das vieldimensionale Spannungsverhältnis ließe sich demnach hypothetisch als Systemkollision zwischen Bildung und Beredsamkeit zuspitzen. Am Ende bliebe ein Dilemma aus Idealismus und Pragmatismus, eine Dichotomie aus der seelesuchenden, aber eher weltflüchtigen Erhebung des isolierten Individuums und der Förderung des so sprachmächtigen wie tatkräftigen Subjekts mit seinem Anspruch auf Gestaltung der Welt eine Dichotomie, die sich über das gesamte 19. Jahrhundert bis in die ihrerseits durchaus offensiven Formulierungen von Thomas Manns Betrachtungen eines Unpolitischen halten würde. Die Förderung einer autonomen Bildung würde damit automatisch auch den Anwendungsbereich der politischen Rede zurückdämmen, da das Individuum als solches ja frei bzw. seine Sprachkunst autonom sein soll. ${ }^{25}$ Beredsamkeit aber bedeutet Eingriff in die Gestaltung der Wirklichkeit,

25 Der Königsberger Philosoph steht gegen die durchaus lange abendländische Tradition eines positiven Nexus aus Rhetorik und Politik, die eine Blüte des Staatswesens nämlich im Gegenteil gerade in Abhängigkeit von der Blüte der republikanischen Gesinnung erkennen will. Stichwort ist die allgemeine Redefreiheit in der öffentlichen Angelegenheit, in der res publica. Hier läge der bedeutende Zusammenhang mit einer nachhaltigen Demokratiekritik bzw. einer folgenreichen Abwertung der Parlamentsrede. Je mehr Rhetorik herrscht, desto weniger besteht die Freiheit des Einzelnen, so lautet die Gleichung, wie sie zwischen Platon und 
Mitwirkung im Staat gemäß dem parlamentarischen Gedanken, in der öffentlichen Sache (res publica) mitzusprechen. Die Freiheit (der Rede) bedeutet in letzter Konsequenz, auch gegen die bestehende Ordnung anzugehen, während die Freiheit des idealistisch Gebildeten darin besteht, sich der freien Einbildungskraft hinzugeben, die den Staat nicht tangiert. Ein somit isolierter Funktionsträger verzichtet aber auf einen argumentativen, also rhetorisch vermittelten Vorbehalt und handelt im Sinne des autoritären Staates.

\section{Institutionalisierung von Bildung im öffentlichen Raum}

Es dürfte sich demnach lohnen, die entsprechenden Vorgänge nun anhand ausgewählter Protagonisten vertieft zu betrachten und entsprechende Indizien, Formulierungen und Konzepte zu sammeln - weniger in den poetischen Werken und großen systemischen Abhandlungen als gerade auch in den pragmatischen Kleinstformen vor Ort: in Paratexten und Unterweisungen, Anordnungen und Regulierungen, im Lehrmaterial selbst oder in der journalistischen Begleitung der Vorgänge durch lokale Medien, natürlich auch in den schriftlichen Korrespondenzen der beteiligten Personen. Die Grundfrage lautet: Welches Wissen wird in welchem Modus an die nächste Generation weitergegeben, wie ist es in das sprachliche Konstrukt eingebunden und welcher Stellenwert kommt in den anvisierten und praktizierten Übertragungsprozessen dabei dem Adressaten zu?

Bevor dies zumindest skizzenhaft geschehen soll, gilt es im Blick auf die stichhaltige These einer rhetorica latens zwei Voraussetzungen zu berücksichtigen: Erstens gibt es nicht die Rhetorik, sondern stets verschiedene, bereits seit der Antike immer wieder kontrovers gewertete Gewichtungen innerhalb eines idealtypischen Systems, etwa zwischen dessen gedanklich-logischen (inventio

Thomas Hobbes auch bereits vor Kant immer wieder in Anschlag gebracht wurde. Die Gefahr, dass einzelne skrupellose Rhetoren das ganze Volk manipulieren und damit in die Geschicke des Staates eingreifen, sucht Kant durch die Betonung des vir bonus zu entkräften, der eben aus natürlicher Veranlagung heraus allein zum Nutzen der Menschen rede und wirke, so dass Wahrheit und Moral sich tatsächlich ohne manipulative Rednerkunst durchsetzen. Nach idealistischer Vorstellung brauchen Aufklärung und Vernunft keine Rhetorik. Bildung darf dann als ,unpolitisch' und damit ,politikfern' und gerade deshalb als ,patriotisch' gelten - die Termini Thomas Manns von 1918 sind hier längst in Kraft - weil der absichtsvoll isolierte Geist mit dem Anspruch auf eine höhere, allgemein menschliche und ewiggültige Wahrheit für die Nation wirkt: im autonomen Bereich der Dichtung. 
und dispositio $)^{26}$ und stilistisch-sprachlichen (elocutio) Anteilen. ${ }^{27}$ Gerade das 18. Jahrhundert unterscheidet mit Wolff, Gottsched oder Baumgarten bereits vor Kant sehr genau eine (eher akzeptierte) „Wohlredenheit“ als herausragendes Sprachgebungsvermögen von der (tendenziell eher abgelehnten) „Beredsamkeit“ als Überzeugungsfertigkeit. ${ }^{28}$ Zweitens aber verschwindet Rhetorik per se niemals oder immer: Jede sprachliche Kommunikation kann unter den Spezifika der rhetorischen Systembildung, also unter den Rubriken inventio, dispositio und elocutio, actio und memoria betrachtet werden. Darüber hinaus liegt es ebenfalls in der systemimmanenten Logik der Rhetorik, dass sie als Kunst wie als Technik eben gerade nicht sichtbar bzw. wahrnehmbar sein darf, also verschwinden muss, um zu wirken. ${ }^{29}$ Und diese besondere Unsichtbarkeit hängt durchaus von der zeitbedingten Wahrnehmungsfähigkeit des Adressaten $\mathrm{ab}$, womit eine diachronische Veränderlichkeit von Form, Praxis oder Wertung zwangsläufig gegeben ist. Rhetorik als intentional strukturierte Sprachäußerung endet also nie, weil sie als Faktur eben nicht verschwinden kann. Lediglich der Wahrnehmungsgrad ihrer Auffälligkeit und Dominanz unterliegt historischem Wandel. Deshalb kann Peter D. Krause auch von einer eher „unbestimmte[n] Rhetorik“ sprechen, ${ }^{30}$ Martin Schierbaum mit gleicher Berechtigung von einer „poetisierte[n]“31 oder Peter Schnyder gar von einer ,magischen“ Rhetorik. $^{32}$

26 Dies führt in letzter Konsequenz bis zur Verselbständigung als „Dialektik“.

27 Dies führt in letzter Konsequenz bis zur Verselbständigung als „Poetik“.

28 Vgl. Kant 1908: § 53, dazu mit Erläuterungen Bezzola 1993: 30-33.

29 Die klassische Rhetorik betrachtet es tatsächlich als die höchste Tugend, sich selbst vollkommen vergessen zu machen, ohne auch nur im mindesten zu verschwinden: es ist die dissimulatio artis, die Wegtäuschung der künstlichen, das heißt allein die Regeln befolgenden Techniken der Redekunst. Die sprezzatura der Rede soll eine natürliche Leichtigkeit vortäuschen, die das mühevolle Fabrizieren strategischer Sprachbastionen unmerklich macht, eben damit der Transfer der Inhalte zum Adressaten umso störungsfreier verlaufen kann. Hier setzt die Kritik Kants an, mit seinem Vorwurf an die Beredsamkeit, die im Gegensatz zur ,ehrlichen“ Dichtkunst stehe. Nach Kant ist aber auch das Kunstwerk zweckmäßig organisiert, ohne dass dabei eine Absichtlichkeit oder Regelhörigkeit erkennbar sein darf, vgl. Kant 1908: § 45. Zu klären bliebe deshalb die konkret noch verbleibende Unterscheidbarkeit von ,kunstvoller Natürlichkeit‘ eines intentionalen Gebildes (Rhetorik) und der ,natürlichen Künstlichkeit‘ des vorgeblich ,absichtslos‘ Gesagten (Dichtung).

30 Krause 2001: 289.

31 Schierbaum 2002.

32 Vgl. Schnyder 1999. 


\section{Beredsamkeit und Bildung in ihrer historischen Tiefendimension}

Vor allem aber wäre die bisher nur skizzenhaft umrissene Systemkollision um 1800 präziser zu fassen, indem auch deren Vorgeschichte ins Blickfeld rückt. Stehen ,Beredsamkeit' und ,Bildung' hier bereits in einem Gegensatz? Seit der Antike ist die Geschichte der Rhetorik mit der Geschichte der Pädagogik sehr eng verbunden: Das oratorische System wurde nie als rein forensisches Wahrheits- oder als politisches Kampfmittel, sondern immer auch als eine reflektierte Schulung des Denk-, Sprach- und Urteilsvermögens verstanden. ${ }^{33}$ Nach antiker, vor allem aber nach frühneuzeitlich-humanistischer Vorstellung ist Rhetorik sogar vorrangig ein Erziehungsmedium, um die junge Generation mit der Systematisierung und der praktischen Anwendung von überliefertem, aber zunehmend auch empirisch erworbenem Wissen vertraut zu machen. Durch Invention und Disposition werden prinzipielle Verbindungen hergestellt und sachlich angemessen dargestellt (inneres aptum) bzw. sprachlich angemessen an den Adressaten vermittelt (äußeres aptum). Rhetorik wirkt somit auch als Technik der Wissensgenese, wobei jedes gewonnene Wissen dann natürlich auch für die jeweiligen Interessen eines Redners eingesetzt werden kann. Das genus deliberativum, die Beratungsrede, ist sogar ausdrücklich dem Zweck der unterweisenden Vermittlung von Informationen verpflichtet, das allgemein menschliche Wissen wird stimmig in die Konditionen des aktuellen bzw. situativen Handelns eingebunden. Als beratende Sonderform tritt hier die geistliche Rede hervor, insbesondere die ermahnende Predigt, wie sie stets - von Melanchthon bis Fichte - entsprechend behandelt wurde. Aber auch poetische Formen wie das moralisierende Drama, die Kasuallyrik oder eine erbauliche Prosa wirken als didaktisch umgesetzte Beredsamkeit, ohne vorsätzlich zu täuschen oder unausweichlich ein einziges Verhalten zu diktieren. Im Gegenteil werden oftmals sogar Alternativen vorgestellt und bewertet, um das Urteilsvermögen (iudicium) des Adressaten zu schulen. Die resultierende eruditio steht seit Philipp Melanchthon zusammen mit scientia (Wissen) ${ }^{34}$ und sapientia (Weisheit) für eine umfassende Bildung im Sinne von Gelehrsamkeit, aber auch bereits für eine ausgeprägte Persönlichkeit, die sich in der konkreten Herausforderung von

33 Fichte diskutiert im Anschluss an Herder die Sprachlichkeit des Denkens und die Denkschulung durch Rhetorik. Vgl. hierzu Bezzola 1993: 96, 98. Auch Humboldt („Über Sprechen und Denken“) geht davon aus, dass der Mensch die einzelnen Sprachoperationen mit den Einheiten seines Denkens gleichsetzt.

34 Im Sinne von Wissenschaft, vor allem ein ,handlungspragmatisch oder technisch anwendbares Wissen“ (Wriedt 2017: 141). 
den Lehrdogmen zu emanzipieren weiß. Im Blick auf die reformatorischen Ansätze Luthers entwickelt Philipp Melanchthon sogar ein eigenes rhetorisches Lehrgenre, das genus didascalicon, dessen Ziel nicht die inhaltliche Überzeugung, sondern allein die Einsicht in einen Sachverhalt ist. Eruditio als rhetorisch vermitteltes systematisches Wissen war in der Frühen Neuzeit jedoch untrennbar mit der pietas, mit Glauben und Frömmigkeit, verbunden.

In der Tradition des antiken Humanismus besteht demnach kein wesenhafter Gegensatz: Beredsamkeit gehört zur (Aus-)Bildung in Schule und Universität, der Redner bildet sich technisch, um moralisch am Gemeinwesen als vir bonus umso sicherer wirken zu können. Hier aber weicht der idealistische Bildungsbegriff um 1800 offenbar ab, da er keine öffentliche Rede in öffentlichen Angelegenheiten, schon gar nicht einen konkreten „Staatsredner ${ }^{\text {‘35 }}$ in der Gegenwart erkennen will (Kant) oder kann (Fichte). Allerdings hatte sich im Zuge der frühneuzeitlichen Territorialisierung bzw. Konfessionalisierung die Figur des streitenden Redners herausgebildet, verbunden mit der Notwendigkeit zur Selbstbehauptung als Subjekt, ganz im Sinne einer engagierten Rednerschaft in eigenem Interesse (homo politicus bei Christian Weise) oder in sachlicher Gegnerschaft zu anderen Interessengruppen. Vor Gericht, in der Politik und in der Glaubenspropaganda verfestigte sich situationsbedingt die Vorstellung des Individuums als eines kämpferischen, defensiv wie offensiv zu sprachlicher Gewalt greifenden Opponenten, der nun eigens dafür ausgebildet werden muss. Nach der Reformation sind es bekanntlich die Jesuitenkollegs, die ihre Zöglinge geradezu abrichten, das erworbene Wissen und jede zu erlangende Kenntnis gezielt zur Schwächung des Gegners einzusetzen. ${ }^{36}$ Hier treten die von Kant beklagten Negativzüge ganz massiv hervor. Erlaubt sind neben der parteigebundenen Formulierung der Wahrheit etwa auch parteidienliche Auslassungen in der Sache, bis hin zu scharfsinnigen Sinnkontraktionen, die sogar zu falschen Annahmen führen können oder als Trugschlüsse gelten müssen. Vor allem ist hier die Kategorie des dubium, des Zweifels, komplett getilgt, da es in Fragen der Rechtgläubigkeit keine diskussionswürdigen Einwände oder gar eine dialogische Sachklärung geben kann bzw. darf. Der Redner soll seinen Adressaten als kognitives wie mentales Subjekt exakt analysieren und diese Kenntnisse dann psychologisch gezielt ausspielen, ja mit komplexen Sprachfiguren, vor allem aber mit stimulierenden Affekten, eine vernunftgeleitete Auffassungsgabe schwächen. Das gesteigerte movere als emotionale Bewegung soll das Gegenüber dann zu einer ursprünglich nicht gewollten Handlung ,bewegen‘. An diesem Punkt setzt

35 Fichte sieht die Predigt in einem direkten Zusammenhang mit einer noch zu erhoffenden politischen Rede in Deutschland, so dass sich der „heutige Kanzelredner in den alten Staatsredner verwandelt: vielleicht!“ (o.A. [i. e. Fichte] 1788: 36)

36 Noch immer das Grundlagenwerk: Bauer 1986; vgl. weitere Hinweise bei Eybl 1998. 
die Aufklärung und vor allem eben Kant mit dem vernichtenden Verdikt an, dass hier neben der unterschwelligen Affektsteuerung durchaus vorsätzliche „Täuschung“ bzw. eine „hinterlistig[e] Kunst“ zu verzeichnen sei, „sich der Schwächen der Menschen zu seinen Absichten zu bedienen“. ${ }^{37}$ Die autonome Position des Adressaten wird im Redeverlauf reduziert, ja eliminiert, er wird zum ausschließlich empfangenden Objekt degradiert. Hier festigt sich eine Vorstellung der rhetorica militans, die tatsächlich mit Suggestion, Emotion und gesteuerter Imagination den Adressaten manipuliert und schließlich überwältigt. Für die Jesuiten erfolgt Rhetorik als ständige Übung und Selbstzucht, die im kämpferischen Einsatz (ecclesia militans) dogmatisches Wissen gehorsam übernimmt, mit darstellerischen Mitteln verstärkt und mit nicht selten sophistischen Techniken unangreifbar macht, um es dann ebenso gewaltsam einem ,mundtot‘ gemachten Adressaten aufzuzwingen.

Auf protestantischer Seite herrschten jedoch nicht weniger Hierarchie und Gehorsam. Allerdings öffneten sich neben der linientreuen Zuarbeit für die Orthodoxie des 17. und 18. Jahrhunderts zunehmend auch poesiegetragene Spielräume für verschiedene Formen der Individualfrömmigkeit. Ausgehend von der evangelischen Betonung des Wortes bzw. dem Argumentationsgebot auf der Basis der Heiligen Schrift in der Muttersprache festigte sich eine Sensibilität für die sprachgetragene Selbstbeobachtung. Verbunden mit der subjektorientierten Gewissensfrage und der hochgewerteten Empirie des Einzelfalls steigerte sich die Selbstwahrnehmung zu einer Form von Individualität, die sich mit der gepflegten ,Innerlichkeit‘ in den protestantischen Pfarrhäusern (Albrecht Schöne) als Gegentradition ausprägte. Nach der formellen Aufhebung des Jesuitenordens 1773 vollzog sich diese Entwicklung - rhetorisch gesehen - dann auch weitgehend konkurrenzfrei. ${ }^{38}$ An die Stelle des tendenziell eher selbstverleugnenden Exerzitiums der Jesuiten im Kollektiv und die monastische Selbstabtötung trat nun, soweit die lokale Orthodoxie es zuließ, eine weltwahrnehmende Selbsterfüllung des Ich, die Kultivierung der eigenen Persönlichkeit. Gegen das Muster von einem dem Kollektiv untergeordneten Subjekt steht damit eine freie, vor allem der Verinnerlichung einer Person verpflichtete Individualität, die natürlich auch mit den entsprechenden sprachlichen Medien auszubilden bzw. zu ,entwickeln' ist.

37 Kant 1908: $327 \mathrm{f}$. Kant sieht die Rhetorik auch als ,Pathologie des Dichtungsvermögens', eine von Sinnlichkeit und Einbildungskraft getragene Rhetorik sei krank, vgl. Hambsch 2007: 157.

38 Es wäre ein interessanter Parallelfall, die von den Jesuiten maßgeblich geprägte Universität Freiburg zu betrachten, die um 1800 ganz eigene, noch durch die Zugehörigkeit zu Habsburg geprägte Reformperspektiven entwarf, vgl. Schiewe 1996. 
Diese Verinnerlichung als Bildungsprinzip, die sich zwischen 1770 und 1810 im deutschsprachigen Raum fest etabliert, greift jedoch auch ihrerseits wieder weit zurück: ein erkennbarer Bezugspunkt sind die Vorgaben der mittelalterlichen Mystiker. Seit Meister Eckharts spirituellen Vorstellungen des göttlichen Bildes, nach dem sich die menschliche Seele aus-bilden bzw. imitierend nach-bilden soll, gilt Bildung als ein innerer und selbstbestimmter Prozess im biographischen Rahmen der einzelnen Person. Schon im 16. Jahrhundert aber wandelt sich diese Auffassung, und neben das theologisch-transzendente Muster treten erste Bestrebungen einer pädagogischen, säkularen Institutionalisierung. Bei Paracelsus heißt es noch in ausgewogener Zweigliedrigkeit, dass der Mensch sowohl aus dem Grundstoff der Welt als auch aus dem Bilde des göttlichen Geistes geschaffen sei. Das Individuum müsse im Laufe seines Lebens gegen teuflische Bedrohungen, vor allem aber auch gegen weltliche Widrigkeiten das Göttliche in sich stärken und ausbilden. Dieser Vorgang aber basiert eben gerade nicht auf aktiver professioneller Didaktik von außen, denn das „Lernen von Menschen ist kein lernen / es ist vorhin im Menschen“. Es geht demnach „allein“ um den auslösenden Vorgang von „erwecken und ermahnen“, ${ }^{39}$ so Paracelsus. Valentin Weigel (1533-1588), Spiritualist und lutherischer Pastor, geht ebenso davon aus, dass „alle Erkenntnis der Wahrheit und Weisheit zuvor im Menschen liege und nicht vom Obiecto, von aussen zu eingetragen werde“. Und entsprechend erfolgt eine maßgebliche Rangerhöhung des eigenaktiven, autonomen und nach Selbstbildung strebenden Individuums - allerdings im festen Bezugsrahmen der Schöpfung, also der kosmischen Ordnung: „Lernen ist sich selber kennen [. . .] du lernst die Welt, du bist die Welt.“40

Im 17. Jahrhundert erstarkte dagegen ein klerikales Normensystem bzw. die entsprechende Buchgelehrsamkeit, vorrangig vertreten durch die lutherische Orthodoxie bzw. deren Institutionen und Hierarchien. Diese beanspruchten das menschliche Wissen formal wie materiell als festen Besitz, um über dessen gesteuerte Weitergabe zu verfügen. Der Bildungsbegriff unterlag hier gänzlich der kirchlichen Administration und damit dem Prinzip der autoritär-dogmatischen, im Grunde aber fortgesetzt scholastischen Belehrung der jeweils folgenden Generation. Anstelle einer transzendentalen Heilsvermittlung stand jetzt die pädagogisch immanente Strukturkonservierung in ausschließlich regenerativer Absicht. Dagegen opponierte wiederum eine entschieden spirituell orientierte Strömung, die dann aufs Neue das Prinzip der Verinnerlichung vertrat. Nach Jakob Böhme ist der menschliche Geist ,der im Spiegel gestaltete Geist Gottes‘,

39 Paracelsus 1590: 423.

40 Weigel 1695: o.P. 
der sich dann im Menschen ,selbst' beschauen kann. Durch die Imagination, durch die intensive Bildkraft des inneren Sehens soll der Mensch dann in der eigenständigen Abkehr vom falschen Weg, nämlich von den Folgen des Sündenfalls, zur Einheit mit Gott finden. Der Mensch kann sich dabei zum Guten wie zum Bösen entwickeln, er besitzt nach Böhme einen „eigenen Willen zur Bildung der Substantz, aus welchem Principio er will“. ${ }^{41}$ Er hat die Freiheit, sich „,zu formen und zu bilden nach seiner Lust und Begierde“. ${ }^{42}$ Aber auch hier besteht eine direkte Bindung an Sprache und Wort: „Gleich wie der Geist der Ewigkeit hat alle Dinge gebildet, also bildets auch der Menschen Geist in seinem Wort.“43 Die Bildung des Worts ist die Bildung ins Wort, das menschliche Wort-bilden muss somit der wortgetragenen Schöpfung entsprechen. Nur so kann der sündhafte Mensch die ihn fesselnde Fleischlichkeit überwinden. Ein analoger Vorgang: Der göttliche Geist bildet alle Dinge (= Schöpfung), der menschliche aber das Geschaffene in das Wort. Die Wortmächtigkeit als Nachvollzug und zeitlich versetzte Wiederholung des göttlichen Schöpfungsaktes obliegt jedem Menschen, allein aus sich heraus und ohne institutionelle Anleitung. Die Gestaltung der Heilsgeschichte durch das menschliche Wort ist behaftet mit der Verantwortung für die Schöpfung, die als ,gebildetes` Individuum konsequent jeder trägt.

\section{Wissen, Bildung und Pädagogik um 1800}

Hier knüpfen nun die Überlegungen um 1800 an. Die Tradition reicht somit von Böhme über Hamann und Herder bis zu Wilhelm von Humboldt, Schleiermacher oder Friedrich Schlegel. Als gemeinsame Bezugsgröße wäre der Begriff von der Bildung bzw. der Kunst als religiöser ,Selbsterlösung“ zu veranschlagen. ${ }^{44}$ Das idealistische Erziehungsideal basiert wiederum auf dem Prinzip der Entwicklung des Einzelnen und betont damit das Prozessuale: Wesen, Person und Individuum sind als Idee und Plan bereits substantiell angelegt und müssen sich im Verlauf der individuellen Biographie zur Sichtbarkeit ,entwickeln', d. h. realisieren. Von außen wird nichts Fremdes hinzugefügt, nur das bereits innerlich Angelegte ist zu enthüllen und zu stärken, immer in einer lebendigen Korrespondenz zwischen beiden Sphären. Dies hätte die neue Universität jetzt vor dem historischen

41 Böhme 1843: 280.

42 Ebd.

$43 \mathrm{Ebd}$.

44 Vgl. hierzu Voßkamp 2004. 
Hintergrund umzusetzen, als Ganzes wie auch in ihren Teilen, also in jedem einzelnen Fach. Demgemäß erklärt beispielsweise der Geograph Carl Ritter 1818 in seiner Einleitung zu dem Versuche einer allgemeinen vergleichenden Geographie, dass es vor allen fachlichen und methodischen Fragen notwendigerweise den „menschlichen Gesichtspunct“ anzusprechen gelte, „um dessentwillen überhaupt sie [die Fachwissenschaft Geographie] nur als wünschenswert erscheint“.

Aber der rechte Wille des Menschen und die Erkenntniß des seiner eigenthümlichen Kraft Entsprechenden in dem außer ihm Gegebenen, so wie die gegenseitige Durchdringung und Steigerung von beiden, diese gehen nur aus dem ernsten Ringen nach der tieferen Erkenntniß des eignen Selbst hervor, und aus der Betrachtung des Menschlichen und alles dessen, was in der Geschichte der Menschheit sich offenbart hat. ${ }^{45}$

Ritter konstruiert hier auch umgehend eine direkte Entsprechung von Individualbildung und Nationenbildung:

Es gehört zum Charakteristischen der menschlichen Natur, daß jedem einzelnen Menschen eine nur ihm angehörige Eigenthümlichkeit einwohnt, durch deren Entwicklung er $\mathrm{zu}$ einem vollkommeneren wird, und so und nicht anders wiederholt sich dies in jedem Volke. In der vollendeten Ausbildung dieser Eigenthümlichkeit liegt die sittliche und mit ihr jede andere Größe des Menschen, wie die Volksthümlichkeit und Nationalgröße der Völker. ${ }^{46}$

Eine vergleichbare Einbindung des Einzelfachs bzw. dessen Bedeutung für die Gesamtheit aller Studierenden zeigt sich in der universalen Aufwertung der antiken Mythologie als einer historischen Orientierungsgröße für den Menschen im Kosmos und damit als unverzichtbare Komponente ,jeder liberalen Erziehung“. Der Altphilologe Aloys Ludwig Hirt (1759-1837) begründet dies:

Aus den ältesten Sagen bildete sich die Volksreligion, und aus dieser die Sitten, die Gebräuche, die Spiele, die Lebensweise. Sie waren die ewige Quelle, aus welcher die Dichter schöpften, und die sie immer in neuen Formen zeigten. Sie lieferten den Stoff zu den ersten rohen Versuchen der Kunst, welche dann, im Gange der Zeit sich fortbildend, jene Mannigfaltigkeit von Idealen schuf, wodurch die alte Welt zum Schauplatz des Schönen und Erhabenen wurde, und deren Überbleibsel noch die Nachwelt mit Bewunderung anstaunt. Aus diesen Gründen betrachtete man die Mythologie von jeher mit Recht als einen nöthigen Theil des Unterrichtes bey jeder liberalen Erziehung; und die Richtung, welche dieses Studium durch neuere Forscher in unsern Zeiten erhalten hat, zeigt die Wichtigkeit und das Anziehende desselben immer mehr. ${ }^{47}$

45 Ritter 1852: 4.

46 Ebd.

47 Hirt 1805: IV f. 
Die Absolutsetzung des Individuums steht also immer noch in größeren Zusammenhängen. Die bei Humboldt aufscheinende Vorstellung von einer lediglich aus der Addition von gebildeten Individuen entstehenden Gemeinschaft wäre mit den geschichtlich überlieferten Kollektivvorstellungen im Sinne einer orientierenden Autorität abzugleichen, etwa mit der wesenhaften Verschmelzung zu einer höheren Einheit im Sinne von ,Nation“ oder gar ,Weltseele‘.

Welcher Status wird hierbei nun der Sprache zugewiesen? Was ist aus Böhmes Benennungskompetenz geworden bzw. jenem allein dem Menschen zugewiesenen Vermögen, die Schöpfung durch seine Sprache zu erhalten bzw. zu erhöhen? Die Selbsterfahrung der Seele als Lernprozess erfolgt auch bei Humboldt mit spezifisch zugerichteten sprachlichen Mitteln. Im Blick auf die rhetorica latens bliebe zu fragen, wie die Rhetorik als eine übergreifende Analyse- bzw. Produktionsfähigkeit auch hier noch in Kraft bleibt. Kann die Universität mit ihren Sprachformen und Textsorten, die die jahrhundertelange Tradition für den akademischen Unterricht bereitstellt, überhaupt ein konkurrenzfähiges Angebot für einen Adressaten machen, der seine umfassende Selbstbildung im Sinne Humboldts oder Goethes eher über die klassischen Genres wie Lehrgedicht, Fabel, Ballade, Erlebnislyrik, vor allem aber über den Bildungsroman selbst initiieren und völlig frei durchführen kann? Hier bieten sich doch außeruniversitär höchst anspruchsvolle und stark nachgefragte Textformate, die dafür sorgen, dass der Adressat in der imitierenden Nachfolge des genialen Dichters eben auch sich selbst ,ausbildet'. Poetische Mittel der Evokation (wörtlich zu verstehen als das Herausrufen aus) oder des wirkungsstarken Fingierens von emotional aufgeladenen Situationen, aber auch Stimmungsschilderungen in der Natur und Landschaft als Stimulus religiöser Regungen (Naturmystik, Pantheismus) bieten höchst authentische Erlebnismomente für den rezipierenden Leser, abgesehen von dramatischen Konstellationen (beispielsweise Verlust-, Entbehrungs- und Wiedergewinnungserlebnis) und dem dispositorischen Ausgleich von Spannungen (Polarität und Steigerung). Diese Möglichkeiten sprechen die Einbildungskraft und das seelische Erlebnisvermögen des Lesers sehr stark an und animieren ihn zur Arbeit an sich selbst, zur Selbsterfahrung der Welt und zur Relativierung des Buchwissens. Wie stark können gerade hier die traditionell gegebenen Unterrichtsformen überhaupt gegenhalten? Können sich in der monologischen Vorlesung, ${ }^{48}$ im monothematischen Lehrbuch oder auch in der mächtigen mehrbändigen enzyklopädischen Fachbilanz überhaupt noch ,selbstbildende‘ Kräfte im Sinne der kosmischen Innerlichkeit entfalten? In den genannten Gattungen herrscht naturgemäß der postulierende Monolog vor, der einen passiven Rezipienten voraussetzt, dessen Einzelerfahrung durch das gesamte Wissen der

48 Vgl. generell Lingelbach 2006. 
Tradition dann eher verdrängt $\mathrm{zu}$ werden droht. Per se ist die Universität in ihrer alten Form ja der Ort der versammelten Fremderfahrung: ${ }^{49}$ Das Wissenskollektiv, das systemische und anwendungsbezogene Zusammenwirken der Fächer in der kollektivierenden und deutlich hermetischen Universitas ließe keinen Raum für die Einzelstimme, das Erlebnisinstitut des Individuums müsste hier eigentlich massiv gefährdet erscheinen. Wie kann es der Universität gelingen, ihre bisherige sprachliche Vermittlungspraxis zu überprüfen und entsprechend Ausgleich zu schaffen? Wie wirkt sich das auf die Verkehrs- als Lehrsprache, auf die Technik der gezielten Adressatenansprache und auf die jeweiligen Gattungen aus?

Zunächst: die Sprache, das konkret eingesetzte Idiom. Im Zentrum von Sprache steht nach Wilhelm von Humboldt „das individuelle Vorstellungsvermögen des Menschen“. ${ }^{50}$ Sprache muss also vertraut machen bzw. als vertraut erkennbar machen, was als Stoff von außen kommt, damit es im Inneren erkannt wird. Sprache muss latent Vorhandenes hervorrufen (Stichwort Evokation) bzw. erwecken. Schon 1780 fasst Fichte es wie folgt:

[D]a feststeht, daß die Keime jeder Wissenschaft und Weisheit in den Geistesanlagen der Menschen eingepflanzt und eingeboren sind, und nichts anderes denn nur die Erweckung derselben erforderlich sei, auf die Art: daß wir die Sache mit unserer eigenen Vernunft selbst durchschaut und aufgefunden zu haben meinen, indem der Meister-Gelehrte dies schaut, damit er uns in die Wege leite, daß auch wir dies finden können. ${ }^{51}$

Der Erfolg dieses Prozesses basiert zunächst auf einem elementaren Wechsel der akademischen Gebrauchssprache: vom traditionellen, aber fremden und historisch fernen Lehridiom der Antike in die Nationalsprache. Fichte plädiert ganz entschieden für die „geschmeidigre $u$. besonders teutsche Mundart““.52 Mit der Ersetzung des Lateinischen durch die Muttersprache wäre eine Individualisierung also zumindest auf der Ebene der Nation vollzogen. ${ }^{53}$ Indem Vorlesungen konsequent auf Deutsch gehalten werden, nähern sie sich idiomatisch dem Erlebnishorizont des

49 Vgl. die verschiedenen Schriften von Friedrich Paulsen über den gelehrten Unterricht oder die deutsche Universität in Paulsen 1912.

50 Humboldt 1994: 24.

51 Fichte 1919: $58 \mathrm{f}$.

52 Fichte 1970: 47-49. Adam Müller sieht die distanzgebietende „Fremdsprachigkeit“ für genauso verderblich an für die Erkenntnisfindung wie die distanzgebietende „Schriftlichkeit“, vgl. Müller 1967: 40.

53 Vgl. die entsprechenden Hinweise zu der bereits von Herder vertretenen Position bei Martyn 2014. Herder betont als Vorteil der Muttersprache die dortige ,Untrennbarkeit‘ von Gedanken und Ausdruck, die uneingeschränkte ,Gewalt‘ über die Worte und das nationale Zugehörigkeitsgefühl via Sprache. 
Alltags an, der Abgleich mit dem Erfahrungshaushalt der eigenen Person wäre stufenlos möglich. Verschärfend hinzu kommt die Ablehnung des Französischen, das noch im 18. Jahrhundert als Kultur- und Diplomatensprache gegolten hatte, aktuell aber für die Sprache des Aggressors steht, der die Existenz des preußischen Staates wie der deutschen Nation insgesamt infrage stellt. Die Wissenschaftssprache entwickelt sich damit in deutlicher Distanz zur Sprache Roms, die im protestantischen Deutschland natürlich längst suspekt geworden war, und zur Sprache des westlichen Hegemonialanspruchs, von Ludwig XIV. bis zu Napoleon I. Entsprechend erfolgen Aktivitäten der Sprachpflege: In der Tradition Luthers und der protestantischen Sprachgesellschaften des 17. Jahrhunderts steht etwa der Sprach- und Sittenanzeiger der Deutschen, den der vaterländische Sprachwissenschaftler Theodor Heinsius (1770-1849) 1817 herausgibt. Heinsius hatte bereits 1814 zusammen mit Friedrich Ludwig Jahn die Berlinische Gesellschaft für Deutsche Sprache gegründet und bittet nun in „Plan und Einleitung“ für den Sprach- und Sittenanzeiger ,die Bewohner aller deutschen Lande[,] durch gegenseitige freie Mittheilung der Gedanken über ihr Heiligstes - ihre Sprache und Sitte“ - fundierte Beiträge zum Erstarken der Nation zu leisten. Auch unter dem Blickwinkel der materiellen Substanzvermehrung der Wissenschaft regt er an, ihm nun aus allen deutschen Regionen entsprechend hilfreiche Materialien, also Untersuchungen, Beobachtungen und Einschätzungen zuzusenden, die der Verbesserung und Pflege der deutschen Sprache dienlich sein könnten, geht es doch um die „Verbesserung der vaterländischen Arbeit“. ${ }^{54}$ Der faktisch vorhandene Pluralismus der deutschen Länder soll als Ressource auch und vor allem für die Optimierung der Wissenschaftssprache genutzt werden. Heinsius bekräftigt hier die Gedanken, die fünf Jahre früher bereits Adam Müller in Wien in einer Reihe von Vorträgen entfaltet hatte: Im siebten Vortrag, Von deutscher Sprache und Schrift, ${ }^{55}$ beschwor Müller den Reichtum der binnendeutschen Diversität, aus der nationales Potential zu gewinnen sei. Eine ausgewogene Dialogkonstellation mit den Regionen ohne eine dominante Kapitale (diese wäre allenfalls rein intellektuell-koordinativ als Bezugsadresse zu sehen, nicht etwa politisch!) unterbreitet geradezu föderale Perspektiven für eine zukünftige Wissenschaft. Müller spricht sich gegen eine „Vermischung“ und für eine „Vermählung“ aus, eine wechselseitige Veredelung im Dialog, die zu einer „dritten“, d.h. „mittleren Sprache“56 führen könnte. Denn das „Allgemeine entsteht nicht durch das Wegwerfen des Besonderen“. 57

54 Heinsius 1817: 1.

55 Müller 1967: 123-126.

56 Ebd.: 126.

57 Ebd.: $123 \mathrm{f}$. 
Keineswegs aber führt die Wendung zur Nationalsprache zu einem automatischen Verzicht auf die Rhetorik.

Wie wäre es, und würde ich mich über diese Dialekte noch beschweren, wenn diese Sprache einen Mittelpunkt hätte, worin sich alle jene Besonderheiten begegnen könnten, [. . .] alle Bildung und alle Unschuld der verschiedenen deutschen Mundarten sich untereinander ausgleichen könnten [...] und alle schroffen Kontraste sich untereinander dämpfen und mildern möchten dadurch, daß sie einem und demselben harmonischen und philosophischen Gesetze untergeordnet würden ${ }^{58}$

Doch der Sprachwechsel allein genügt nicht: Neben dem Idiom des Adressaten wäre es die konkrete Adressierungsstruktur, die glaubhaft zu machen hätte, dass es tatsächlich um die Entwicklung des Individuums geht. In der gezielten Anrede zeigt sich der respektgetragene Anspruch gegenüber dem Lernenden, und der didaktische Redner hätte diese sprachlichen Leistungen überzeugend zu erbringen. ${ }^{59}$ Gilt der Adressat als ein zu unterweisender Schüler, der ohne Kenntnisse in der Sache antritt und folglich mit fremden Inhalten zu beladen ist, oder als das zu bildende Individuum, das alles zu Lernende latent bereits in sich trägt, das nach dem platonischen Anamneseprinzip dann gemeinsam sprachlich zu aktivieren wäre? Fichte zielt bereits 1780 auf eine performative Wiederholung des Erkenntnisaktes:

[M]ein Vortrag ist immer synthetisch; ich werfe meine Gedanken nie hin, wie ich sie unsichtbar in meiner Studirstube gedacht haben, sondern ich denke sie, finde sie, entwikle sie vor den Augen der Hörer, und mit ihnen; ich bemühe mich dabei den strengsten logischen Gang auch in den kleinsten Theilen des Vortrags zu gehen [...] durch Vorzeichnung des Ganges der Spekulation an einer Tafel. ${ }^{60}$

\section{Ethische Apologie und didaktische Praxis von Rhetorik nach 1800}

Vor allem scheint die scholastische Aufbereitung von Sachverhalten und die präskriptive Klassifikation auf dem Prüfstand zu stehen, insbesondere in Gestalt

58 Ebd.: 123.

59 Auch hier steht eine rhetorische Kernkompetenz (äußeres aptum) dahinter, verbunden mit attentum parare (Offenheit) und tua res agitur (Betroffenheit).

60 Also neben auditiver auch visualisierte Sprache, vgl. Fichte 1970: 60 f. Zu verweisen wäre auch auf die entsprechenden Positionen Schellings (1802), hier läge bereits der Ansatz, etwa die Vorlesung als Lehrmedium und Vermittlungsform zu optimieren. 
der monologischen Kathederrede. Die entsprechenden Debatten wären auf der Ebene einer Metagattung zu verfolgen, nämlich in der ,Lehre über die Lehre‘. Natürlich stehen hier zunächst die großen Systemschriften Fichtes, Schellings, Schleiermachers oder Humboldts zur Betrachtung an, aber einige wenige Stichproben können bereits zeigen, dass man sich gerade auch in weniger prominenten Kreisen höchst versiert und engagiert mit den anliegenden Aufgaben der didaktischen Kommunikation beschäftigt. ${ }^{61}$ Ein auffälliger Befund vorweg: Die Rhetorik als Lehrgegenstand ist aus dem akademischen Curriculum faktisch verschwunden. Lehrveranstaltungen zur Redekunst sind zumindest bis 1834 äußerst selten. Die Altphilologen bieten sporadisch einzelne Übungen zu Quintilian an, aber auch nur über das X. Buch der Institutio Oratoriae, das die griechisch-römische Literaturgeschichte allein im Zusammenhang mit stilistischen Fragen rekapituliert. Schlecht besuchte Veranstaltungen ${ }^{62} \mathrm{zu}$ Demosthenes, Isokrates, Thukydides oder Cicero zeugen von einer deutlichen Randständigkeit der rhetorischen Lehre als solcher. Im Praxisbereich begegnet einzig die akademische Festrede, die ebenso ausschließlich in die Zuständigkeit der Altphilologie fällt und bis 1848 durchweg auf Latein gehalten wurde. Bemerkenswert ist dagegen die breit gefächerte und durchweg anhaltende Konjunktur gedruckter Lehrwerke zur Verbesserung der persönlichen Sprach- und Darstellungskompetenz. Diese zeigen deutlich das Bestreben, Beredsamkeit und Bildung in einem komplementär gedachten Gleichgewicht zu halten, das von ethischen wie formalen Aspekten geprägt ist. Das geht schon aus den Titeln hervor: 1807 publiziert Ludwig Purgold (1780-1821) Gedanken Über die Bildung zur Poesie und Beredsamkeit auf Schulen, 1831 formuliert Karl Justus Blochmann propädeutisch Ein Wort über die Bildung unserer Jugend zur Wohlredenheit und öffentlichen Beredsamkeit. Beide setzen bereits auf der gymnasialen Stufe an. 1836 schließt Wilhelm Wackernagel seine Academischen Vorlesungen zu Poetik, Rhetorik und Stilistik an. Darüber hinaus steht über Jahrzehnte eine breite Palette praktischer Hilfsmittel zum Selbststudium bereit: Titel wie Die deutsche Beredsamkeit in der Ausübung oder: Sammlung deutscher Muster zur Bildung eines deutschen Redners (anonym 1780) stehen neben einer Mustersammlung für die Declamationsübungen der Jugend (anonym 1818) oder Christian Friedrich Falkmanns Hülfsbuch der deutschen Stylübungen (1822) bzw. dessen Methodik der deutschen Stylübungen (1823). Seine Praktische Rhetorik (1831) bietet auch eine Vorrede für die Schüler mit einer

61 Inwieweit die betreffenden Werke als Popularisierung der prominenten Texte oder als originäre Leistung zu werten sind, bliebe zu untersuchen.

62 Vgl. Viermond 2011. 
ausgearbeiteten „Abfassungslehre“ und „Vortragslehre“. 1831 sorgt ein Rhetorisches Wörterbüchlein für terminologische Klärung.

Exemplarisch $\mathrm{zu}$ betrachten wäre in diesem Zusammenhang der bereits genannte Theodor Heinsius. In Der Redner und Dichter oder Anleitung zur Redeund Dichtkunst (1810) entscheidet er bereits im Titel die Frage einer Systemkollision mit auffallender Eindeutigkeit, indem er beide Sphären parallel ordnet ohne Hierarchie und ethische Wertung. ${ }^{63}$ Er geht davon aus, dass Redner wie Dichter neben den „besonderen Naturanlagen“ vor allem eine „nicht gemeine wissenschaftliche Bildung“ benötigen, weshalb schon die Schulen die Fächer Rhetorik und Poetik gleichermaßen anzubieten hätten. ${ }^{64}$ In einer „Philosophie der Rhetorik“ unterscheidet er „verschiedene Seelenkräfte der Sprache und der Rede“, nämlich das „Vorstellungs-, Gefühls- und Begehrungsvermögen“. ${ }^{65}$ Darüber hinaus erfolgt eine wichtige Begriffsbestimmung der „Beredsamkeit“ in einer Darlegung zweier genereller Ansprüche des Menschen: Entweder gilt es, „auf die Gegenstände der Außenwelt einzuwirken, oder: sein eigenes Gemüth im Hoffen und Glauben an das unendlich Wahre, Schöne und Gute zu erheben“. ${ }^{66}$ Was für Kant in der qualitativen Wertung noch unvereinbar schien, definiert Heinsius kurzerhand als zwei anthropologisch gleichberechtigte Komplementärbereiche. Die Sprache steht neutral als Grundkompetenz für beide Anwendungen bereit: einmal strebt ihre Kraft nach außen, auf das handlungsbezogene Vorstellungsvermögen (Sprache des Verstands), einmal nach innen, auf das Empfindungsvermögen (Sprache des Gefühls). In systematischer, nicht aber in wertender Absicht trennt Heinsius Poesie und Prosa, ${ }^{67}$ während die Letztere wieder zu unterteilen sei in einen theoretischen und einen praktischen Zweck, der eben jeweils in der Erkenntnis (Vorstellungen) wie auch in der Handlung (Begehren) liege. Um den höheren Zweck zu erreichen, nämlich „das Begehrungsvermögen in Thätigkeit zu setzen, oder den menschlichen Willen zu Handlungen zu bestimmen“, muss die Zusammenführung des „Vorstellungs- und Gefühlsvermögens für einen Gegenstand“ gelingen. ${ }^{68}$ Beides soll also verbunden und ausgewogen sein, deshalb haben sich prosaische und poetische Darstellungskunst einander anzunähern. Aus ihrer

63 Vgl. auch Adam Müller zur Gleichrangigkeit von Poesie und Beredsamkeit im Bild der „Ehe“, was auf gegenseitige Hilfe und fruchtbare Wechselwirkung verweisen soll, Müller 1967: 88-90.

64 Heinsius 1810: vii ff. (Vorrede zur ersten Ausgabe).

65 Ebd.: $1-5$.

66 Ebd.: 6-10.

67 Vgl. ebd.: 5.

68 Heinsius 1810: 6. Vgl. zum Gefühlsaspekt der Wissenschaft auch bei Savigny und Schleiermacher Treß 2012: 188f. bzw. 192. Fichte vertrat schon 1780 eine Rhetorik, die auf einer ,Lehre von den eingeborenen Gefühlen` basiert. Die rhetorischen Regeln sind allgemein-anthropolo- 
Vereinigung bildet sich dann offenbar etwas Drittes, die „Sprache der Beredsamkeit““ ${ }^{69}$ Damit formuliert Heinsius ein integratives pragmatisches Synthesemodell, das keine Komponente ausgrenzt und vielmehr eine entsprechend wechselseitige Steigerung in Aussicht nimmt. ${ }^{70}$

Hier könnte sich der Ansatz für eine schulpraktische Unterweisung in Sachen einer idealistischen Beredsamkeit zeigen. Heinsius löst das Dilemma kurzerhand auf: Jedes Individuum soll pragmatisch handeln (auf die Gemeinschaft einwirken) und gleichzeitig innerlich reflektieren (auf das eigene Gemüt einwirken) und sich selbst dadurch nach idealistischen Kriterien bilden. In der sich anschließenden praktischen Betrachtung bietet der Autor dann neben den klassischen Teilen des rhetorischen Systems eine Gattungstypologie, die sich u. a. auch sehr eingehend der „beschreibenden“"71 und der „didaktischen“72 Poesie widmet und hier insbesondere die „allegorische Poesie“ mit Fabel, Parabel und Allegorie würdigt. Dann aber folgt als „,die eigentliche didaktische“ die „gnomische Poesie“ und das „eigentliche Lehrgedicht““ ${ }^{73}$ Damit ist für ein breites begriffliches Spektrum nebst ganz konkreten Hinweisen an den didaktischen Redner gesorgt, der seinerseits immer auch über poetische Mittel verfügen darf, um den Adressaten dann mit besonders anschaulicher Darstellungskunst zu eigenem Handeln im wissenschaftlichen, nicht zuletzt aber auch im politischen Raum zu ermutigen.

Für die entschiedene Bekräftigung einer moralisch fest eingebetteten Rhetorik als Vermittlungskunst sorgt dann 1814 (bzw. in zweiter Auflage 1837) der Theologe Franz Theremin mit seinem Buch Die Beredsamkeit ist eine Tugend, oder Grundlinien einer systematischen Rhetorik. Für den protestantischen Pastor gilt naturgemäß die Predigt als zentrale Textsorte, ${ }^{74}$ er formuliert seine Konzepte aber durchaus mit einem gattungsübergreifenden Gültigkeitsanspruch im idealistischen Sinne: „Das höchste Gesetz der Beredsamkeit, anwendbar auf alle Verhältnisse, worin der Redner sich befinden kann, hat in dieser Schrift den Ausdruck erhalten: Er suche seine jedesmalige Idee auf die beim Zuhörer nothwendig innewohnenden Ideen zurückzuführen [. . .].“75 Mit der idealistischen Formel

gisch und stimmen mit den ,geistigen Naturanlagen“ des Menschen , auf das passendste“ überein (Fichte 1919: 33).

69 Heinsius 1810: 7.

70 Damit könnte auch eine Motivation zum sprachlichen Handeln im politischen Raum gemeint sein, die aber zumindest im Preußen des frühen 19. Jahrhunderts keine Resonanz findet.

71 Heinsius 1810: $216 \mathrm{f}$.

72 Ebd.: $251 \mathrm{f}$.

73 Ebd.: 251-267.

74 Vgl. Conrad 2012: 25-29, 139-147, 162-164.

75 Theremin 1837: ivf. 
der „innewohnenden Ideen“ reformuliert Theremin die traditionelle Verpflichtung des Rhetors auf das äußere aptum: Jedem Menschen sind a priori idealtypische Orientierungsmuster gegeben, die der Redner kennen und im Blick auf seinen Redeerfolg berücksichtigen muss. Da es sich um anthropologische Konstanten handelt, kann der Redner diese natürlich auch an sich selbst erkennen: „[U]nd dies Gesetz, wie ebenfalls bemerkt worden ist, kann er nur erfüllen, wenn die sittlichen Ideen kräftig und lebendig in ihm wohnen. Diese Ideen in ihrer Gesammtheit sind der Wille Gottes, und der durch ihn dem Menschen, und namentlich dem Christen, vorgezeichnete Beruf.“76 Prädestination und göttlicher Heilsplan setzen einen festen Rahmen, in dem der Redner allerdings nach seiner eigenen Einschätzung wirken darf: Die Wortwahl im Einzelnen bleibt ebenso ins Belieben gestellt wie der Aufbau der gesamten Rede, diese hat etwa keiner strengen Geometrie oder formalen Disposition zu folgen. Damit reduziert Theremin deutlich ein mögliches systemphilosophisches Übergewicht der Sache im Sinne des inneren aptum zugunsten des allein adressatenorientierten äußeren aptum:

[M]an hat, da die Idee in keine so bestimmte Schranken eingezwängt ist, eine größere Freiheit in den Wendungen, und wird durch keine Rücksichten auf Symmetrie verhindert, die Gedanken, die die wichtigsten sind, auch am meisten auszuführen, und einen jeden da hinzu stellen, wo er am kräftigsten erscheint. Es wäre jedoch wohl zu bemerken, daß man hier zwar scheinbar regellos, aber deshalb nur um so geregelter einherschreiten, und nicht minder streng als sonst den Hauptgedanken festhalten, und die Vorstellungen, die ihn ins Licht setzen, auswählen müßte. ${ }^{77}$

Rhetorisch gesprochen darf der Verfasser sich auf die Lizenzen des ordo artificialis berufen und in Sachen dispositio die größtmögliche Freiheit walten lassen, sofern er die sprezzatura achtet und „scheinbar regellos“ verfährt.

In seinem 4. Kapitel kommt Theremin dann zum titelgebenden Problem und löst es kategorisch: „Die Beredsamkeit ist eine Tugend“. Eigennutz oder Fremdbestimmung via Rhetorik stellen im Rahmen einer allgemein gefestigten Ethik keine Gefahr dar:

Da aber alles Wirken des Menschen in seinen Verhältnissen, unter der Leitung des Sittengesetzes steht oder stehen soll, so kann die Ausübung der Beredsamkeit, da sie nichts andres ist als ein solches Wirken, auch keinen anderen Gesetzen unterworfen seyn, als den ethischen. Sie strebt in den Gesinnungen und der Handlungsweise anderer Menschen eine Veränderung hervorzubringen; die Frage nach ihren Principien verwandelt sich also

76 Ebd.

77 Ebd.: xxvii. 
ganz natürlich in diese: welches sind die Gesetze, nach welchen ein freies Wesen auf andere freie Wesen wirken darf? und diese Frage kann nur aus der Ethik beantwortet werden. $^{78}$

Alle aus der „Rhetorik als Theorie der Beredsamkeit“ abgeleiteten Regeln sind ethische Regeln und die „Theorie der Beredsamkeit ist also ein Teil der Ethik““. ${ }^{79}$

Das 5. Kapitel („Von den Ideen“) thematisiert die Frage der Adressatenansprache, indem Theremin nun ganz ausdrücklich das „Verhältniß“ betrachtet, „worin sich der Redner mit dem Zuhörer befindet““. ${ }^{80}$ Ersterer hat Pläne und Absichten, die er aber zunächst gegen „die Trägheit der gleichgültigen Gemüther“ aufseiten der Rezipienten durchsetzen muss, um diesen einen „Anstoß“ zu geben, „wonach sie handeln“ sollen. Ferner muss er auch die

offenbar widerstrebenden besiegen und mit sich fortreißen. Es steht ihm aber durchaus kein Zwangsrecht über die Gemüther zu; er ist kein Gesetzgeber, der die Verhältnisse der Menschen ordnet, und sie so auf eine mittelbare, aber sichere unwiderstehliche Weise lenket; kein Herrscher, der ein ganzes Volk hier oder dorthin führet, weil er über Eigenthum, Leben und Ehre eines jeden gebietet. Er steht denen ganz gleich, auf die er wirken will. ${ }^{81}$

Die Doktrin der Gleichrangigkeit untersagt dem Redner jegliche, sowohl offene als auch unterschwellige, paternalistische Verfügung über den Adressaten. Er muss dessen „Freiheit achten“ und darf selbige nicht etwa durch die Erregung von Leidenschaften oder gar „Bethörung“ des Verstandes einschränken. Der Wille des Redners wie der des Angesprochenen müssen sich entsprechen. Eine einsichtige Ausführung des Gesagten muss die Folge sein. Der Hintergrund für eine solche gewaltfreie Kongruenz von Willen, Einsicht und Akzeptanz ist wiederum die idealistische Auffassung, dass alle Menschen etwas „ganz Allgemeines und Nothwendiges“ wollen und ihrer „sittlichen Natur nach“ wollen müssen, „dadurch daß die wahre Freiheit des Menschen stets nach der Realisirung gewisser Ideen strebt, die sich berechnen und deutlich aufzeigen lassen. [...] Denn so wird die Wirkung des Einen auf den Andern, die Freiheit des letztern nicht aufgehoben, und dieser erfüllt nur auf fremden Antrieb, was er aus innerem Trieb stets zu erfüllen trachtet. “82 Das Konzept der platonischen Erweckung allgemeinster Prinzipien schließt Fremdbestimmung per se aus. Mit dem Gleichheitsgrundsatz überwindet Theremin die Kant'schen Vorbehalte gegen den Sprecher als Hegemon und den Hörer

78 Ebd.: 22.

79 Ebd.: 23.

80 Ebd.: 26.

81 Ebd.

82 Ebd.: $27 \mathrm{f}$. 
als unterjochtes Subjekt: „Ideen überhaupt sind produktive Gedanken, die zum Hervorbringen und Handeln antreiben, und selbst der Keim des Hervorzubringenden, so wie die Regeln der Form sind, die es erhalten soll."83 Die ethischen Ideen, die bei jedem Menschen in seinem Vernunftbegriff liegen, müssen angesprochen, also mit sprachlichen Mitteln aktiviert werden, damit sie wirken und die entsprechende Handlung nach sich ziehen können. Die höchste ist die „Pflichtidee“, gefolgt von der „Tugendidee“ und der „Glücksidee“, die durch fortlaufendes ideengerechtes Handeln verwirklicht wird. Der Redner muss lediglich für die „Belebung“ dieser Ideen beim Adressaten sorgen, um ihn „zum höchsten Grad des Bewußtseyns und der Selbstständigkeit“ zu erheben. ${ }^{84}$ Das alles liest sich einerseits wie eine idealistische Apologie der Rhetorik, um sie vor ungerechten Vorwürfen als Täuschungsinstrument zu schützen, andererseits aber auch als Appell an alle Anwender, die oratorische Kunst verantwortungsbewusst einzusetzen.

\section{Adressatenansprache als performative Gattung: Mündlichkeit, Dialog und Gespräch}

Auf dieser Grundlage einer idealistisch verankerten und ethisch legitimierten Beredsamkeit konkretisiert der bereits mehrfach genannte Theodor Heinsius seine Überlegungen für ein entsprechend adressatengerechtes Bildungserlebnis. Unter der Rubrik „Die Lebhaftigkeit“ diskutiert er die „Figuren der Aufmerksamkeit“, die Funktion von „Phantasie“, „Witz“ und „Scharfsinn“, aber auch die Rolle der unterschiedlichen Gemütsbewegungen. Vor allem liegt ihm an den eigenartigen „Formen des Gesprächsstyls“ unter der Notwendigkeit des „Lehrstyl[s]““ ${ }^{85}$ Die entsprechenden Hinweise zum „mündlichen Styl“ beziehen sich aber gerade nicht auf die Deklamatorik des Einzelredners, sondern auf einen lehrhaften Dialog im Rahmen des akademischen Unterrichts: Gesprächsstil als Lehrstil. Zwar kannte schon die klassische Rhetorik die Stilfigur der vorweggenommen Gegenrede des Adressaten, die als fester, vom Autor selbst formulierter Bestandteil einer Rede zugehörte. Die Prolepsis (oder anticipatio) ist aber rein fiktiver Natur, bedeutet also keineswegs den ergebnisbestimmenden Einwurf eines realen Zuhörers. Sie bleibt Kalkül des monologisierenden Akteurs. Die reale Mitwirkung am Erkenntnisprozess des Adressaten ist damit also noch nicht ermöglicht. Zwei

83 Ebd.: 28.

84 Ebd.: $29 \mathrm{f}$.

85 Heinsius 1810: 114. 
Jahre nach Heinsius sollte Adam Müller diesen Aspekt mit einer Opposition aus stummer Dichtkunst und lebendiger Mündlichkeit noch verstärken. Gemäß einer gemeinschaftsbildenden Funktion weist er ihm sogar eine nationale Dimension zu. Bei Müller erschöpft sich die Beteiligung des Publikums jedoch in der inspirierenden Unterstützung, indem es an dem Gesagten reaktiv Anteil nimmt und den weiteren Fortgang mit Impulsen zwecks weiterer „Verfertigung der Gedanken“ beim Reden mitbestimmt. ${ }^{86}$ Müller formuliert eine Theorie des produktiven Dialogs und betont vor allem den Nutzen der konträren Meinung für eine gemeinsame Erkenntnisvermehrung: Das „Gegenteil“ gilt es nicht nur formal anzuerkennen, sondern auch sprachlich aktiv nachzuvollziehen zwecks Annäherung an das Ganze. ${ }^{87}$ Andere Autoren flankieren diese Position auf ihre Art, Wilhelm von Humboldt etwa handelt in seinem Vortrag Über den Dualis über ,Geselligkeit“ und ,Sprache': ${ }^{88}$

Alles Sprechen ruht auf der Wechselrede, in der, auch unter Mehreren, der Redende die Angeredeten immer sich als Einheit gegenüberstellt. Der Mensch spricht, sogar in Gedanken, nur mit einem Andren, oder mit sich, wie mit einem Andren, und zieht danach die Kreise seiner geistigen Verwandtschaft, sondiert die, wie er, Redenden von den anders Redenden ab. Diese, das Menschengeschlecht in zwei Classen, Einheimische und Fremde, theilende Absonderung ist die Grundlage aller ursprünglichen geselligen Verbindung. ${ }^{89}$

\section{Schon die Sprache an sich ist Humboldt zufolge}

kein blosses Verständigungsmittel, sondern der Abdruck des Geistes und der Weltansicht der Redenden; die Geselligkeit ist das unentbehrliche Hülfsmittel zu ihrer Entfaltung, aber bei weitem nicht der einzige Zweck, auf den sie hinarbeitet, der vielmehr seinen Endpunkt doch in dem einzelnen findet, insofern der einzelne von der Menschheit getrennt werden kann. ${ }^{90}$

86 Ganz im Sinne der unmittelbaren Kontaktaufnahme mit einem gegenwärtigen Publikum auch im Sinne der ,Geistesgegenwärtigkeit‘ Gottes, der durch den Mund des Redners spricht, vgl. Peter Schnyder im vorliegenden Band. Laut Müller erlebt Deutschland derzeit ein „stummes Jahrhundert“, während er das britische Parlament als einen „Schauplatz wahrer Beredsamkeit“ preist (Müller 1967: 118), auch „heiliger“ Beredsamkeit dank Edmund Burke (ebd.: 120).

87 Vgl. Kapitel „II. Vom Gespräch“ in Müller 1967: 87.

88 Humboldt 1907: 25. Vgl. dazu Burkhardt 1987; Lorenz 1973; umfassend: Hösle 2011.

89 Humboldt 1907: 25.

90 Ebd. 
Auch Fichte ${ }^{91}$ und Schelling werben, wie erwähnt, mit dem „genetischen Stil“92 für eine dialogisch orientierte Lehre, ebenso Schleiermacher, der für das Entwickeln der Inhalte ,vor den Augen“ des Adressaten plädiert und damit nicht zuletzt an das rhetorische Instrument ex evidentia anknüpft. ${ }^{93}$ Der Hofprediger Theremin bekräftigt 1837 in seinem Beredsamkeitsbuch durchweg die dialogische Methode, die er anstelle eines systematischen Vortrags zu praktizieren wünscht. In der Systemkonkurrenz mit dem Bildungsroman gälte es in Parenthese allerdings auch zu fragen, was etwa ein Dialog in diesem Medium tatsächlich bedeutet. Faktisch bleibt er ja ein Monolog des Dichters, der seine Meinung in den Figurengesprächen lediglich auf zwei Personen verteilt. $\mathrm{Zu}$ fragen wäre, bis zu welchem Grad hier ein echter Diskurs entsteht, ein lebhafter Disput mit ungelösten Dilemmata, eine Kontroverse, die eben zwecks Aktivierung des Lesers auch ungelöst im Raum stehen bleiben könnte? Gewinnt dagegen nicht die Universität einen wirklichen Vorteil, weil der Rezipient selbst, physisch präsent, den Gang des Dialogs mitbestimmen, also auch umlenken kann? Im Bildungsroman tritt der Autor nach seinem festen Plan und seiner festen Absicht hinter seine Figuren, als ein Exeget und letztlich Moderator, der harmonisiert, damit aber auch den Erkenntnisgang letztlich in seinem (Autor-)Horizont beendet. Ein Leser bleibt passiver Rezipient.

Hier gilt es sich nochmals Franz Theremin zuzuwenden, der im Jahre 1836 vor dem Hintergrund einer langjährigen Berliner Hochschulerfahrung - eine Schrift mit dem Titel Über die deutschen Universitäten. Ein Gespräch vorlegt. Die „Einleitung“ thematisiert Bedrohliches: „Die Jünglinge, welche die deutschen Universitäten besuchen, sind auf denselben stets großen Gefahren ausgesetzt gewesen, die sich in der letzten Zeit noch deutlich vermehrt haben.“ Aktuell mit Reformen anzusetzen, erschiene jedoch bedenklich, weil „diese Institute in ihrer jetzigen Gestalt zu sehr mit dem Leben und den Neigungen der Deutschen verflochten sind“. Grundsätzlich habe „von der Wissenschaft selbst [...] der Antrieb zu einem sittlichen Leben“ auszugehen. ${ }^{94}$ Im Rekurs auf das idealistische Bildungskonzept, das auf die gesamte Persönlichkeit ziele, droht dem Studierenden laut Theremin vor allem eine konkrete Versuchung: dass er im falschen Glauben an seine persönliche Ehre dem gefährlichen Duellierungswahn verfalle. Damit begibt er sich nämlich außerhalb des bergenden Rechtsrahmens der Universität: Studentische Verbindungen können auf diese Weise sein Verhalten als Individuum fremdsteuern und destruktiv beeinflussen. Mit Blick auf

91 Fichte handelt in Deduzierter Plan einer höheren Lehranstalt (entstanden 1807) über ,dialogische Formen“ (Fichte 1817: § 7).

92 Oesterreich 1997: 451.

93 Vgl. den Beitrag von Ernst Müller im vorliegenden Band.

94 Theremin 1836: 23. 
die programmatische Diskursförderung im Gespräch muss das Duell natürlich als fatale Verkehrung der bilateral gesteigerten Bildung erscheinen: Die Konfrontation wird nicht verbal, sondern durch die physische Vernichtung des Gegenredners ,geklärt‘. Theremin fordert hinsichtlich der Bildung einer sittlichen Persönlichkeit eine entsprechende Einhegung und unterbreitet einen Vorschlag, dessen Umsetzung nur „mit geringen Schwierigkeiten verbunden wäre, und von welchem sich für die wissenschaftliche Ausbildung und für die Sittlichkeit der Studierenden die heilsamsten Wirkungen erzielen ließen“. ${ }^{95}$ Vor allem formuliert Theremin seine Idee gleich in der praktischen Umsetzung: Auf 40 Seiten präsentiert er ein fiktives programmatisches Gespräch zwischen vier älteren Herren, die auf ihr eigenes Universitätsleben bereits zurückblicken. Der euphorische Reformer Adalbert disputiert mit zwei behäbigen, aber lernfähigen Skeptikern, Ludovico und Theophilus. Der Reformer spricht im Namen des Autors das Wundermittel aus und entkräftet gleich in dessen Vollzug die kritischen Gegenreden seiner Freunde, so lange, bis diese keine Einwände mehr haben. Der Vorschlag lautet in den Worten Adalberts wie folgt:

Das Mittel wäre, daß die Professoren das Geschäft des Denkens nicht allein übernähmen, sondern es mit ihren Zuhörern theilten; daß die geistige Thätigkeit des Lehrers seine Schüler zu einer ähnlichen Thätigkeit heranzöge; und daß zu diesem Ende die monologische Form des Vortrags oft in die dialogische überginge. ${ }^{96}$

Das Ziel ist hier tatsächlich, Bildung als Prozess weitestgehend auf die studierenden Individuen selbst zu übertragen: „Man würde nicht ein so großes Pensum absolvieren; aber man würde die Selbstthätigkeit der Studierenden erwecken, und sie würden dann leicht das Fehlende ergänzen. “97 Der bloße „Vortrag des Lehrers“ dagegen besitze „keine hinlänglich anregende Kraft“, und nur mit der dialogischen Form könne „ein Funken wissenschaftlicher Begeisterung“ in das „Innere“ der Studierenden fallen, sie ,entzünden“ und somit zu eigenständigen Leistungen befähigen. $^{98}$,Funke‘, ,Entzündung‘ und ,Begeisterung“ können in ihrer vordergründigen metaphorischen Funktion kaum darüber hinwegtäuschen, dass es sich hier um eine explizite Wiederaufnahme mystischer Leitbegriffe handelt. In Rücksicht auf Hierarchie, Autorität und Fremdbestimmung als Rahmenbedingungen der Lehre liegt also eine eindeutige Präferenz der Autonomie und Selbstverwirklichung vor, dem verbalen Widerpart kommt aber eine wichtige impulsgebende Assistenzfunktion zu.

95 Ebd.: 10.

96 Ebd.

97 Ebd.: 11.

98 Ebd. 
Gegen die kleinlichen Einwände seiner Partner stellt Adalbert dann deutlich den unabweisbaren Vorteil der Methode heraus:

Weil der wissenschaftliche Sinn Selbstthätigkeit ist, weil ein einziger Gedanke, den ich in mir selbst hervorbringe, mehr Werth hat in Rücksicht auf meine Bildung, als tausend Gedanken, die ich von einem andern in mich aufnehme; und weil diese Thätigkeit für die Mehrzahl der Studierenden durch das ruhige, passive Anhören nicht erregt, sondern ertödtet wird. ${ }^{99}$

Nicht ohne ironischen Unterton spielt Adalbert dann auf jene bei seinen Freunden offenbar fehlende Erfahrung eines dialogisch aktivierenden Unterrichts an: „Oft ist während der Universitätsjahre der Geist verdunstet, und es ist nur ein Phlegma zurückgeblieben, welches, keiner Begeisterung, keiner eigenen Ideen = Erzeugung fähig, sich nur in einem mechanischen Geschäfts = Gange gefallen kann, ja vielleicht selbst für diesen keine Tüchtigkeit besitzt.“100

Theremin hält in dieser eingängigen Gesprächsszene ein Plädoyer für ein aktivierendes Unterrichtsgespräch in Form eines vorbildlichen Sachdialogs, der durchaus fordert und sogar provoziert: Adalbert setzt mit seinen Partnern genau das anschaulich und prototypisch um, was sein Autor für die Reform des Unterrichts vorschlägt: eine adressatenaktivierende Bildungsrhetorik. Dies richtet sich gegen langweiligen Monolog ${ }^{101}$ und ermüdende Kanzelrede wie auch gegen den allwissenden Erzähler als Autorinstanz im Bildungsroman. Die Autorisierung des Adressaten hat Vorrang, die Übertragung der anteiligen Mitwirkung am Lernprozess als Bildungsprozess ist eine prägende Selbsterfahrung, auf die der Studierende im Blick auf seine sittliche Reife, aber auch im Blick auf seine eigene Erkenntnisfähigkeit und damit auch auf mögliche eigene Forschung nicht verzichten kann. Statt passiv „,von Anderen Gedachtes in sich auf[zu]nehmen“, soll nun die Fähigkeit erworben werden, „selbst zu denken“. ${ }^{102}$

Natürlich tritt auch hier wiederum das bewährte Medium des philosophischen Dialogs hervor, wie es als didaktisches Instrument bei Plato begründet liegt, in der Neuzeit u. a. bei Hutten, Harsdörffer, Thomasius, Diderot, Hume oder Mendelssohn praktiziert wurde und sich schließlich auch um 1800 großer Beliebtheit

99 Ebd.: 9.

100 Ebd.: $18 \mathrm{f}$.

101 Kontrovers diskutiert stehen hier zwei didaktische Mittel: das Gespräch gestattet Einwürfe, spontane Neuorientierung und plötzliche, inspirierte Zusätze. Die Vorlesung galt dagegen jedoch als langweilig, man war zum Schweigen und Stillsitzen verurteilt und sah sich als Nachschreiber den schlecht vorbereiteten Ergüssen eines Einzelnen ausgesetzt. Das traf nicht nur auf die Vorlesungen Hegels zu. Als sittliche Folgen wurden Stumpfsinn, Verweigerung, Faulheit und Schwänzereien beklagt.

102 Theremin 1836: 8. 
erfreute: Bruno oder über das göttliche und natürliche Prinzip der Dinge (1802) zeigt sich als der Versuch Schellings, eine allgemeinverständliche Darlegung seines philosophischen Systems (Identitätsphilosophie, Ich und Natur), mit den Techniken der Wechselrede zu formulieren. Philipp Weber benennt die spezifischen Wirkungsqualitäten, etwa „lose Regeln des Absetzens, improvisierte Anschlüsse und willkürliche Abfolgen“, und stellt darüber hinaus einen Zusammenhang mit dem Typus des „Begriffsdramas“ (Alain Badiou) her, in dem nun aber anstelle der auftretenden Personen eigentlich die von ihnen genannten und in der ständigen Wiederaufnahme zunehmend differenzierten Begriffe als Akteure hervortreten. Das philosophisch abstrakte Thema findet sich somit „in einen dezidiert kontingenten Zusammenhang gestellt“ und wächst darin als „kommunikatives Ereignis“. ${ }^{103}$ Bezüge zeigen sich auch zu Schleiermachers Theorie des geselligen Betragens (1799/1800), wo das Gespräch als „freies Spiel“ der Kräfte gilt. ${ }^{104}$ Seine „Dialektik“105 bezeichnet Schleiermacher selbst noch als eine ,kunstmäßige Gesprächsführung im Gebiet des reinen Denkens‘. Wie Humboldt die Wissenschaft als permanenten Suchprozess umschreibt, so formuliert Schleiermacher mit seinem dialogischen Prinzip einen dynamischen Wissenschaftsbegriff, der in einem deutlichen Gegensatz etwa zur Systemphilosophie Hegels oder Schellings steht. Theremin zielt aber noch viel stärker auf die authentische, von physischer Koaktion getragene Vermittlungstechnik, die eine reale Wechselrede anstelle der literarischen, also schriftlich vorgegebenen fordert. An die Stelle des monologischen Frontalvortrags eines hierarchisch höher gestellten Dozenten setzt der Verfasser eine lebhaft antagonistisch agierende Performanz mit beiderseitigem Wissensgewinn.

In Theremins szenischer Verlebendigung seiner Bildungstheorie ist gleichzeitig aber auch, so unauffällig wie deutlich, die Wiederkehr der Beredsamkeit bzw. ihr ungebrochener Vollzug zu erkennen: In rhetorischer Terminologie handelt sich hier schlicht um eine sermocinatio, die Wiedergabe von Thesen durch fiktive Figuren, die mit Hilfe der Einbildungskraft möglichst authentisch zu gestalten sind und vom Rezipienten mit entsprechendem Vertrauenskredit aufgenommen werden. Speziell handelt es sich um die Prosopopöie, in der abstrakte Begriffe anschaulich, nämlich redend und handelnd, figuriert werden. Theremin praktiziert dies in der Tat mit einem deutlich poetisierenden Duktus: Er fingiert eine Realität und überzieht diese mit markanten anekdotischen, ja zotischen und durchaus

103 Weber 2017: 77.

104 Vgl. dazu Schmid 2006.

105 Als Vorlesung gehalten 1811, vgl. die Bemerkungen in der entsprechenden Einleitung des Drucks 1833: Schleiermacher 1988: 117. 
zeitkritischen Zügen des Biedermeierlichen, ohne dabei aber die Brisanz des Gegenstands aus den Augen zu verlieren. Der Diskurs erfolgt damit stark ästhetisiert: Atmosphärische, sinnliche und sentimentale Werte animieren das Sensorium des Adressaten und steigern den Übermittlungserfolg. Der Vergleich etwa mit Wilhelm Raabe oder dem späteren Theodor Fontane liegt nahe, es ist die Poesie, die hier der Wissenschaft als Vermittlungsmedium dient. Aber es handelt sich keineswegs um eine Imitation des Romans als Fiktionsinstrument, sondern um ein im Kern entschieden traditionelles rhetorisches Standardmittel, um gerade das Abstrakte oder Sperrige für einen Adressaten rezipierbar zu machen.

\section{Grundlegung einer Erfolgsgeschichte deutscher Universität und Wissenschaft}

Damit aber offenbart sich bereits eine epochale Differenz zu den frühen Jahren um 1800: Nach Demagogenangst, Reaktion und zunehmend gefestigter Restauration erfolgt im Jahre 1836 ein energischer Widerstand gegen eine allzu liberale Unterrichtspraxis und die ,Mitsprache' des Adressaten im wörtlichen Sinne. Außerhalb der Fiktion des Theremin'schen Dreiergesprächs pochte man nämlich ganz energisch auf die Beibehaltung der alten Form des Monologs, möglicherweise auch aus Besorgnis darum, eine entsprechend kritische Stimmenvielfalt im politischen Raum einhegen zu können. In einer Entgegnung auf Theremins Thesen äußert sich der Rezensent (E. A.) in der Jenaischen Allgemeinen LiteraturZeitung vom April 1836 wie folgt:

Seit den letzten zwanzig Jahren haben sich so viele literärische Aerzte - bald Allöopathen, bald Homöopathen - zur Behandlung der Universitäten aufgeworfen, daß man zuletzt glauben könnte, die Universitäten litten wirklich unter einer schlimmen Krankheit. [...] Die allerunglücklichsten Versuche neuerer Zeit waren aber die, wodurch man den Urcharakter der Universitäten - das rein wissenschaftliche Streben und die systematische Bildung der Köpfe - in blosse Abrichtungsanstalten für den künftigen Broterwerb und Staatsdienst verwandeln wollte. Dahin gehört denn auch der Vorschlag des Herrn Theremin, den er im vorliegenden Dialoge [. . . ] macht, auf den Universitäten, statt des systematischen Vortrages, die dialogische Methode einzuführen. Abgesehen davon [...], wie ein Professor in Vorträgen vor mehr als hundert unterzeichneten Hörern, die er nicht einmal nach ihrer individuellen Bildungsstufe persönlich kennt, das Systematische und Dialogische verbinden soll, um doch im Laufe eines Halbjahres eine in sich fest begrenzte Wissenschaft gleichmässig beendigen zu können; [...] was würde durch die dialogische, nach Tertia, und höchstes noch nach Secunda der Gymnasien gehörende, Schulform gewonnen? Vielleicht die leichtere Auffassung und Durchbildung irgendeines wissenschaftlichen Systems? Die klare Vergegenwärtigung des nothwendigen inneren Zusammenhanges aller 
seiner Theile? Oder soll eben der streng wissenschaftliche Charakter der Studien dadurch beseitigt werden? Würden solche durch Dialoge und Examina abgemüdete Schwachköpfe noch Sinn für den Ernst der Wissenschaft behalten? - Haben seit Jahrhunderten die Universitäten ihre große Aufgabe ohne solche Schulmeistereyen und Kleinigkeitskrämereyen befriedigend gelöset; sind sie selbst, einst verkannt, angefochten und theilweise in ihren wesentlichen Rechten verkümmert, der Stolz der deutschen Nation geblieben, und ist mit ihnen kein Universitätswesen des Auslandes zu vergleichen; warum ununterbrochen an ihnen ändern und hofmeistern ${ }^{106}$

Offenbar missversteht der Rezensent die Positionen Theremins absichtsvoll und wirft ihm eine blinde Autoritätshörigkeit („Abrichtungsanstalt“) bzw. eine prinzipielle Verachtung von Wissenschaft vor, zu der er seinerseits mit Kernbegriffen wie ,Reinheit‘, ,Ernst‘, ,Strenge‘ oder ,feste Begrenzung ein markantes Verhältnis pflegt. Er unterstellt, dass durch die Beteiligung und die Eigenaktivierung des Schülers ein Übergewicht des Unwissenden entstünde und dass der qualifizierte Unterricht einer Koryphäe ,zerredet‘ würde. Der platonisch gedachte Dialog erscheint entstellt zum ermüdenden Prüfungsgespräch, so dass nichts als kleingeistige Schulmeisterei bliebe, ganz im Gegensatz zur Vorlesung, die ein eigenes, von Nachfragen ungestörtes Mitdenken im Horizont des „Ernst[es]“ der Wissenschaft gestatte. Der anonyme Kritiker steht hier nachweislich nicht allein: Aufgrund eines strittigen Erlasses des Kulturministeriums entzündete sich zu dieser Zeit eine heftige Debatte um die vorlesungsbegleitenden oder auch gänzlich eigenständigen ,konversatorischen Übungen‘. Von den Gegnern wurden diese als lediglich abprüfende Fragereien abgewertet, die unter Abschweifungen und Unterbrechungen den Gegenstand zerfaserten, wogegen die konzentrierte monologische Vorlesung zu preisen sei, die Wissen geordnet, durchdacht und im Zusammenhang stimmig zu vermitteln verstehe. ${ }^{107}$

Für das Prinzip der Vorlesung als auditives Verfahren der Wissensvermittlung spricht unabhängig davon auch noch ein elementarer Zusammenhang zwischen Phonetik und Geistesübertrag, den bereits Jakob Böhme unter mystischen Voraussetzungen konstatierte und auf den sich wiederum Herder, Humboldt oder Hegel berufen: ${ }^{108}$

Dann mit dem Hall oder Sprache zeichnet sich die Gestalt in eines andern Gestaltnis ein / ein gleicher Klang fänget und beweget den andern / und im Hall zeichnet der Geist seine eigene Gestaltnis / welche er in der Essentz geschöpffet hat und hat sie im Principio zur

106 E. A. 1836: $37 \mathrm{f}$.

107 Vgl. Spenkuch 2010, insb. Kap. 2.4: „Die Kontroverse um konservatorische Übungen im Lehrbetrieb (1843-1848)“.

$108 \mathrm{Zu}$ spezifischen Fragen des Hörens und zum „Phonozentrismus“ bei Herder, Hegel und Humboldt vgl. Trabant 1990: 169-184. 
Form gebracht / daß man im Wort verstehen kann / worinnen sich der Geist geschöpffet hat $[\ldots]^{109}$

$\mathrm{Zu}$ fragen bliebe abschließend nach der weiteren Durchsetzung bzw. nach dem Anteil der beiden strittigen Methoden an der nun zur Jahrhundertmitte deutlich einsetzenden Erfolgsgeschichte der deutschen Universität. Offenbar besteht die wahre Systemkonstellation nicht zwischen Bildung und Beredsamkeit, sondern zwischen einer monologischen, autoritären Systemphilosophie und einer eher liberal verstandenen dialogischen Erkenntnisfindung mit Bevollmächtigung des Adressaten, in jeden vorgetragenen Sachverhalt auch konstruktiv einzugreifen oder dortige Ansätze und Argumentationskonstrukte kritisch umzuwerten. Die Frage scheint nicht die nach „Beredsamkeit“ oder „Wohlredenheit“, Kunst oder Natur, Rhetorik oder Poesie, momentaner Manipulation oder ewiger Wahrheit zu sein, sondern vielmehr die nach dem zugebilligten Mitwirkungsradius des Angesprochenen: In welcher Form wird er auktorial in den Erkenntnisgang mit eingebunden? Der dialogisch-deliberative Ansatz zielt ja durchaus darauf ab, dass der Studierende sich als eine Persönlichkeit erkennt und festigt, die selbst zur Wahrheit findet und sich damit auch selbst bildet, vor allem aber darauf, dass er am vorgeführten Erkenntnisprozess nicht nur anteilig mitwirkt, sondern diesen bald im Transfer auch ohne jede fremde Hilfe an jedem anderen beliebigen Gegenstand initiieren kann.

Unter der nationalen Prämisse wäre nun Anlass gegeben für die Vermutung, dass hier vielleicht eine spezifisch deutsche Schwerpunktsetzung erfolgt: ${ }^{110}$ auf der Basis der Tradition eines mystischen und damit eigenaktiven Bildungsverständnisses wird der Hörer also zum Sprecher in der Gestalt, dass er dann auch selbst gegenüber dem Objektbereich des Geistes wie der Natur zum Forscher werden kann, der in einen eigenständigen Dialog mit der Transzendenz, mit Gott zu treten versteht. Gestärkt wäre der Mut zur eigenen Beobachtung, die dann zur eigenen Position berechtigt und in der Kombination mit Konklusion und Progression ein neues wissenschaftliches Prinzip initiieren dürfte. In der $o b$ jektiven Perspektive aber resultiert aus dem dialogischen Prinzip eine nachhaltige Dynamisierung von Wissen: Wissensgewinnung erfolgte als Prozess, der in der alternierenden Folge von spezifizierenden Gesprächsbeiträgen eine graduelle Steigerung aufwiese. Gegen die Statik einer unveränderlichen Wissenssystematik (Systemphilosophie und Scholastik) träte die Rhetorik dann als Instrument der antithetischen Spannungsgestaltung und erzeigte sich damit als Grundprinzip der modernen, auf inhaltliche Progression ausgerichteten

109 Böhme 1718: 1676 (Anhang Cap. I).

110 Vgl. Bollenbeck 1996. 
Wissenschaft. Das Aushandeln in Rede und Gegenrede führt zu einem zu Anfang noch nicht bekannten Ergebnis, das beide Vorgaben, nämlich die jeweils nur temporär gültigen Positionen der Dialogpartner, ,aufhebt‘. Festgefügte dogmatische Schemata der Wissenssystematik verlieren dadurch ihre Verbindlichkeit, Wissenszuwachs und Innovation erscheinen möglich.

In der Kombination einer spirituellen Selbstvertiefung als Subjekt mit der dynamischen Objektivation der Erkenntnis im Wechselgespräch könnte dann eine deutsche Wissenschaftstradition entstehen, die anstelle des Brotgelehrten, des lediglich reduplizierenden Wissensverwalters, auch den Typus des inspirierten Genies in der akademischen Wissensgenese etablieren würde. Die Mystik als nationales Kapital erfährt hier ihre Transformation in den Modus der technischen Erfindung bzw. der naturwissenschaftlichen Entdeckung. Ein derart in der Sache gestärkter Typus ließe den deutschen Forscher dann, so wäre eine Hypothese zu formulieren, als Koryphäe im Wettbewerb der Nationen möglicherweise dominanter auftreten. Die mittelalterlichen Vorgaben der mystischen Individualität bzw. die Selbstinbezugsetzung zu Größen wie Gott oder Natur wurden durch die Erfahrungen im jesuitischen wie im preußischen Untertanenstaat stark zurückgedrängt und erschienen um 1800 dann nur noch im Ausgrenzungsbereich der ästhetischen Autonomie als scheinbare Freiheit. Der wissenschaftliche Fortschritt im Sinne des grenzüberschreitenden Erkenntnisgewinns aber lebt ja gerade von der Figur des aufbegehrenden Einzelgängers im ,faustischen Streben', der schließlich aber auch der Gemeinschaft, dem Staat, zu helfen vermag: Von beengenden Prinzipien entbunden und durchaus wagemutig verstößt er gegen metaphysische wie säkulare Erkenntnisverbote und schafft damit neues Wissen. Ganz in der Vorstellungstradition eines verbotenen, aber erfolgversprechenden Bündnisses mit höheren Mächten vollzieht der faustische Entdecker ohne hemmende Skrupel graduelle wie prinzipielle Fortschritte im Wissenserwerb. Er agiert als kalkulierender Innovator wie als inspirierter Impulsgeber, ${ }^{111}$ so dass der Genius des Wissenschaftlers in Analogie mit dem des ,originellen“ Dichters zu setzen wäre. Das ,Originalgenie‘ wäre ganz entsprechend auf die Wissenschaft zu übertragen. An diesem Punkt wirken Gemeinschaft, Dialog und Rücksichtnahme auf Bestehendes eher negativ, als Resultat eines temperierenden, moderierenden ,Mittelns` stünde Mittelmäßigkeit, wogegen die elitäre, herausragende und alles umkehrende Impulskraft des ,Geniestreichs', also der Regel- wie Konventionsbruch (,gegen alle bisher geltenden Annahmen') stünde. Wie stark ist die optionale Ermittlung von Wissen gewährleistet

111 Zufall, Eingebung oder Traum als vorgeblich irrationale Größen haben in der Naturwissenschaft und der dortigen Erkenntnisbildung durchaus ihren Platz, worauf etwa die einem Traumbild folgende Entdeckung der Struktur des Benzolrings verweist, wie der Chemiker August Kekulé in einem Selbstprotokoll berichtet. 
bzw. das Recht, gegen kollektive Überzeugungen und Denkstile eine begründbare Einzelmeinung zu setzen? In welchem Umfang sind die Möglichkeiten garantiert, den Wahrheitsdiskurs aktiv und selbstbestimmt mit einer produktiven bzw. progressiven Forschungsdynamik zu führen, auch mit vorläufigen Vermutungen, mit Spekulation und Zweifel? Der Raum für zumindest vorübergehend gültige Wahrheiten muss in der Wissenschaft wie in der Politik gegeben sein, bis sie dann einem gemeinschaftlichen Prüfprozess ausgesetzt und graduell durch empirische wie sprachliche Verifikation bzw. Falsifikation als Wahrheit proklamiert werden. Seit Descartes jedoch werden (dynamische) Rhetorik und (statische) Wissenschaft als getrennte Systeme betrachtet. ${ }^{112}$

Alle genannten Aspekte münden damit in die noch offene Problematik, welchen Stellenwert die Rhetorik nun nach 1800 einnimmt. Von Kants Offensive gegen die Beredsamkeit gehen tatsächlich wegweisende Impulse für die programmatischen Grundsatzfragen des 19. und 20. Jahrhunderts aus. Dahinter stellt sich ganz konkret die Frage nach der Wirkung des Monologischen wie nach den Praktiken des Dialogischen: Wie stark wirkt Rhetorik als autoritär vorgetragene geistige Einzelleistung und wie stark als ein mehrsträngiges Gespräch? Dies wäre vor allem in den oben genannten Bezugsbereichen zu fragen, in der Theologie, der Politik und der Pädagogik. In welcher Weise erwachsen hier Diskursformen, die dem Individuum eine aktive Mitwirkung via Rede und Gegenrede gestatten, so dass in der Konsequenz auch von einer dynamischen Pluralisierung von Glaubensbekenntnis, Staatswohl oder Wissenschaft die Rede sein könnte? ${ }^{113}$

112 Die Problematik ist also nicht zu vorschnell einem einseitigen Wertungsschema einzupassen, etwa das dialogisch-inspirative Modell (mit quasi demokratischer Adressatenbeteiligung) als Zukunft und Fortschritt und das monologisch-systemphilosophische (mit autoritär-hegemonialer Adressatenunterdrückung) als Vergangenheit und Rückschritt. Das erfolgreiche Staatswesen lebt dialektisch von beiden Anteilen: Die militärisch wie bürokratisch straffe Verwaltung garantiert Stabilität durch Gleichordnung, die innovative Findungspotenz des souveränen Subjekts stellt dynamisierende Konzepte zur Verfügung. Hier erfüllt der ,Bildungsroman' als Sedativum die notwendige Aufgabe, das Subjekt als möglicherweise aufbegehrenden Träumer oder einen in der Phantasie Vagabundierenden zu disziplinieren, vgl. Hegels despektierliches Wort von der Heranbildung des ,Philisters“ im Roman.

113 Verknappt gesagt würden sich Rhetorik und Religion im Laufe des Jahrhunderts in Entsprechungzum preußischen Monarchismus zunehmend verhärten zu einem affirmativen Hof- und Staatskirchentum, als ,religiöser Dialog، bliebe nichts als Kulturkampf und Antisemitismus. Auf dem Gebiet der politischen Redekunst aber, wie sie Fichte schon im 18. Jahrhundert von Deutschland erhoffte, begegnen außerhalb Preußens Frühformen der Parlamentsrede, die in ihren indikatorischen Qualitäten zwischen 1848 und 1918 große Schwankungen aufweist. Adam Müller begründete es im Vorfeld: „Darum gedeiht in Republiken die Beredsamkeit, nicht bloß weil jedem mitzureden erlaubt ist, sondern weil jeder frühe gewöhnt wird, einzugehn in 
Für die moderne Wissenschaft läge hier die besondere Herausforderung, das graduelle Verfahren von Beobachtung und Beurteilung eines Gegenstandes derart in eine Formulierung zu überführen, dass nicht nur die elitäre fachliche Gegenrede erfolgen kann, sondern dass auch die potentielle Mitsprache aller anderen Disziplinen in der Universitas, vor allem aber auch der gänzlich außerhalb der akademischen Forschung stehenden Kreise möglich wird. Gefordert ist damit die formale Beachtung einer breiten publikumsorientierten Verständlichkeit, ${ }^{114}$ was neben Gliederung und Folgerichtigkeit primär auf die Ebene der stilistischen Kunst (elocutio) zielt. Ganz im Sinne des Geniebegriffs hätte der Wissenschaftler nicht nur auf dem Felde seiner fachspezifischen inventio begabt zu sein - das Ergebnis bliebe im Sinne eines gemeinschaftlichen Nutzens wirkungstaub -, sondern auch und gerade als beredter Multiplikator seiner Einzelleistung in Hinsicht auf die studierende Hörerschaft, die gesamte Nation, ja auch die Weltöffentlichkeit schlechthin. Bildung durch Wissenschaft könnte somit die traditionellen Institutionen und ihre Eingrenzung verlassen und sich auf alle Interessenten, insbesondere auch auf den noch unvorbereiteten Nachwuchs ertragreich auswirken. Den somit gefragten Typus verkörpert ideal der Chemiker Justus Liebig, erhält er doch schon 1854 seine entsprechende Zertifikation durch niemand Geringeren als die führenden Köpfe der deutschen Nationalphilologie: „die chemie kauderwelscht in latein und deutsch, aber in Liebigs Munde wird sie sprachgewaltig“. ${ }^{115}$ So bescheinigen ihm Jacob und Wilhelm Grimm in ihrem Deutschen Wörterbuch seine auch und gerade stilistisch überzeugenden Spitzenleistungen. Das macht Schule: mit Theodor Mommsen bekommt der „gegenwärtig größte lebende Meister der historischen Darstellungskunst“ im Jahre 1902 sogar den Nobelpreis für Literatur. In seiner Römischen Geschichte (1854-1856) offeriert der Historiker zwar eine faktenbasierte Objektivität, durchsetzt diese aber ganz stark mit den wirkungsorientierten Mitteln der subjektiven Imagination. Wie selbst erlebt treten dem Leser die historischen Personen etwa als stark psychologisierte Individuen und profilierte Akteure vor das innere Auge. Der Adressat nimmt teil an dramaturgisch durchkomponierten Szenen und sorgsam disponierten Dialogen, ${ }^{116}$ erlebt die eindringliche Gegenwärtigkeit der Räume und Landschaften. Prosopopöie bzw. sermocinatio als Technik

die freie Gesinnung, in das Ohr des Nachbars, weil, wer herrschen will, so vieles Unabhängige, so viel eigentümliche Weise zu hören und zu empfinden, neben sich dulden muß, und so vielen gehorchen muß.“ (Müller 1967: 75)

114 Von Christian Garve (Garve 1796) bis zu modernen Konzepten des popular science writing öffnet sich ein weites diachronisches Spektrum.

115 Grimm/Grimm 1854: XXXI.

116 Also zeigt sich hier wiederum das Prinzip das Theremin'schen Modells eines glaubwürdig fingierten Gesprächs, das als quasi selbst gehört gewertet werden kann. 
der sprachlichen Evokation wahrer oder auch erdichteter Personen ex evidentia bestechen mit größter Lebensechtheit, so dass der Leser glauben darf, sie leibhaftig vor sich zu sehen. Eine syllogistische Beweisführung wechselt mit sorgsam kalkulierten Abschweifungen, Ausschmückungen oder Auslassungen. Pathos und Ethos sorgen in sensibler Ausgewogenheit für Aufmerksamkeit und Akzeptanz. All das geht jedoch weit über das traditionelle attentum parare hinaus. Der oratorisch geschulte Geschichtsschreiber zieht hier sämtliche Register des Systems und generiert virtuos mit allen sprachlichen Mitteln eine überzeugende Wirklichkeit - und damit weitestgehend Wahrheit. Die elitäre Forschung steht nun endgültig in einem unausgesprochenen Pakt mit der autonomen Dichtkunst zwecks Steigerung einer effizienten Wissensvermittlung. ${ }^{117}$ Stillschweigend geht die Entwicklung damit über Kants Versagen hinweg, der die wissenschaftliche Substanz einer komplexen Systemphilosophie mit seinem ,Packpapierstil‘ (Heinrich Heine) eben nicht zu kommunizieren und die Wissenschaft also nicht in den Dienst einer sich in Aufklärung befindlichen Menschheit zu stellen vermochte. ${ }^{118}$

Ein galoppierender Anstieg der populärwissenschaftlichen Formate schon seit 1800 ist die Folge. Von Johann Beckmann, Johann Heinrich Helmuth oder Christian Garve (Von der Popularität des Vortrags, 1796) bereits angestoßen, verstärkt dies wiederum Adam Müller 1812, auch hier unter dem Rubrum der „Geselligkeit“:

Indes sind auch solche öffentlichen Vorlesungen über Gegenstände der Kunst vor einer Versammlung von Personen, die weniger die Absicht, zu erlernen oder Kenntnisse zu erkaufen, als ein allgemeines, wahrhaft menschliches oder gesellschaftliches Interesse an den Fortschritten der Bildung vereinigt, förderlich für die Belebung unserer Sprache, und überhaupt eine neue, ehrenwerte Gattung in Deutschland. Auch der erste, der wissenschaftliche Teil unserer Literatur, will also endlich gesellig werden. ${ }^{119}$

Damit ist eine breite nationale wie internationale Wirkung der deutschen Wissenschaft gesichert, nicht zuletzt auch im Blick auf die Rekrutierung des so notwendigen Nachwuchses. Gedankliche Tiefe, empirische Beglaubigung und stilistische Eingängigkeit verbinden sich $\mathrm{zu}$ einem äußerst erfolgreichen

117 Noch Wilhelm von Humboldt unterscheidet die ,Tätigkeit der produktiven Einbildungskraft in Poesie, Philosophie und Geschichte‘ als ,rednerischen Gebrauch der Erkenntnis‘ von einem ,wissenschaftlichen‘ Gebrauch, vgl. Zöllner 1989: 66.

118 Kant, der Aufklärer, wäre demnach ,konservativ' weil elitär ausgerichtet: das Wissen bleibt Wenigen vorbehalten. Adam Müller erscheint dagegen ,progressiv` da er ein parlamentarisches Prinzip der allgemeinen Mündlichkeit nach dem Idealtypus des britischen Parlaments installieren will. Er zielt auf die Gemeinschaft und sucht den Ausgleich mit den außerakademischen Laien, ein Geben und Nehmen der ,Provinzen“ mit der ,Hochsprache‘

119 Müller 1967: 39. 
Produkt, das langfristig exportfähig ist. Wo aber die Ausbildung zum Wohlredner oder gar die Begabung als Dichter nicht vorhanden ist, erwächst Hilfe in Gestalt des fachgebildeten Schreibers, der dem Fachvertreter gerne zu Diensten steht. Als prominenter Prototyp begegnet hier Wilhelm Bölsche, der mit seiner populären Darstellung von biologischem Wissen in der Nachfolge Ernst Haeckels Massenauflagen erzielt. In seinem Buch Die Naturwissenschaftlichen Grundlagen der Poesie (1887) betrachtet er die Produktion (inventio) und Übertragung (elocutio) von Wissen ganz modern in den monistischen Mustern der Naturwissenschaft und homogenisiert, wie bereits vor ihm Émile Zola, auch den Poeten mit dem Experimentalforscher:

Der Dichter [...] ist in seiner Weise ein Experimentator, wie der Chemiker, der allerlei Stoffe mischt, in gewisse Temperaturgrade bringt und den Erfolg beobachtet. Natürlich: der Dichter hat Menschen vor sich, keine Chemikalien. Aber [...] auch diese Menschen fallen in's Gebiet der Naturwissenschaften. Ihre Leidenschaften, ihr Reagieren gegen äussere Umstände, das ganze Spiel ihrer Gedanken folgen gewissen Gesetzen, die der Forscher ergründet und die der Dichter bei dem freien Experimente so gut zu beobachten hat, wie der Chemiker, wenn er etwas Vernünftiges und keinen wertlosen Mischmasch herstellen will, die Kräfte und Wirkungen vorher berechnen muss, ehe er ans Werk geht und Stoffe kombiniert. ${ }^{120}$

Diese ,Chemie“ jedoch ist längst bekannt und seit der Antike bis in ihre feinsten Substanzen reflektiert bzw. begrifflich etikettiert: eben mit der klassischen Rhetorik. Bei Bölsche klingt in der gewollten Strukturanalogie einer regelgeprägten Poesie mit der exakten Abbildlichkeit einer wissenschaftlichen Naturwahrheit eine idealistische Beredsamkeit mit modernstem Antlitz an, ${ }^{121}$ die streng der Programmatik des Naturalismus verpflichtet ist. Eine identische Logik herrscht in der Natur, in der Anthropologie und in der Dichtung.

Die Frage nach einer Systemkollision zwischen Beredsamkeit und Bildung um 1800 muss damit unausweichlich zum Befund einer Aufwertung der Rhetorik als verborgener Leitdisziplin auch im 19. Jahrhundert führen. Vielleicht liegen hier die Gründe für die verschleiernde Hilflosigkeit in der Begriffsfindung seitens einer Forschung, die zu klären versucht, ob und wie Rhetorik nach der Aufklärung verschwunden sein könnte. Dagegen stünde der befreiende Ansatz, dass Rhetorik eben immer bleibt und immer maßgeblich auf allen Feldern menschlicher Existenz beteiligt und als vielgestaltiges Instrument auf die Wissens-, Glaubens- und Entscheidungspraxis gerichtet ist. In Deutschland wächst

120 Bölsche 1887: 7.

121 Vor allem aber öffnet Bölsche auch dem Zweifel breiten Raum: der Leser wird angehalten, eigene Bedenken auch gegen das elitäre Wissen zu erheben. 
im 19. Jahrhundert die zunehmende Abwehrhaltung gegen (Kants) Systemphilosophie samt deren hermetischer Imprägnierung durch eine unkommunikative Sprache. Es geht gegen die elitäre Verschließung von Wissen gegenüber der Allgemeinheit und gegen die Trennung des sprachbegabten Individuums von der gesellschaftlichen Realität durch einen idealistisch isolierenden Bildungsbegriff. Positiv aber geht es um eine dynamische Offenheit des Wissenserwerbs, um eine schrankenlose Teilnahme aller durch das Angebot einer allgemeinverständlichen Wissenschaftssprache, die als ausgleichende Kombination aus alltagsnahem Dialekt und distanzierender Hochsprache zwischen Abstraktion und Anschaulichkeit einen umfassenden Erkenntnisfortschritt erlaubt.

$\mathrm{Zu}$ lange also wurde die Rhetorikforschung vom Kant'schen Imperativ auf die Frage von Wahrheit und Täuschung eingeengt; ertragreicher wäre, zu klären, welche Ansätze einer historisch wirksamen Diskurspraxis sich jeweils zeigen. Hier könnte der Blick frei werden auf die phänomenologische Vielfalt der Einsatzbereiche im Gegensatz zur idealistischen Verengung, die letztlich auf die Ausgrenzung einer nichtautonomen Sprachkunst in öffentlichen Angelegenheiten zielt, damit aber den neuralgischen Strang der pädagogischen, theologischen und politischen Kultur des 19. und 20. Jahrhunderts zu tilgen versucht.

\section{Literatur}

Arndt, Andreas (2013): Geselligkeit und Gesellschaft. Schleiermachers Versuch einer Theorie des geselligen Betragens. In: Ders., Friedrich Schleiermacher als Philosoph. Berlin, Boston: De Gruyter, 51-63.

Bauer, Barbara (1986): Jesuitische „ars rhetorica“ im Zeitalter der Glaubenskämpfe. Frankfurt a.M.: Lang.

Bezzola, Tobia (1993): Die Rhetorik bei Kant, Fichte und Hegel. Ein Beitrag zur Philosophiegeschichte der Rhetorik. Tübingen: Niemeyer.

Blochmann, Karl Justus (1831): Ein Wort über die Bildung unserer Jugend zur Wohlredenheit und öffentlichen Beredsamkeit, womit zur dießjährigen Prüfung der Zöglinge seiner Erziehungsanstalt und des Vitzhum'schen Geschlechtsgymnasiums hochachtungsvoll und ergebenst einladet Karl Justus Blochmann. Dresden: Meinhold.

Böhme, Jakob (1718): Einleitung zum wahren und gründlichen Erkenntnis des grossen Geheimnisses der Gottseligkeit [. . .]. Hrsg. von Nicolaus Tscheer. Amsterdam: Wetstein.

Böhme, Jakob (1843): Mysterium Magnum oder Erklärung über das erste Buch Mosis. Hrsg. von Karl Wilhelm Schiebler. Leipzig: Barth.

Bölsche, Wilhelm (1887): Die Naturwissenschaftlichen Grundlagen der Poesie. Leipzig: Reissner.

Bollenbeck, Georg (1996): Bildung und Kultur. Glanz und Elend eines deutschen Deutungsmusters. Frankfurt a.M.: Suhrkamp.

Bornscheuer, Lothar (1989): Rhetorische Paradoxien im anthropologie-geschichtlichen Paradigmenwechsel. In: Rhetorik 8, 13-42. 
Burkhardt, Armin (1987): Der Dialogbegriff bei Wilhelm von Humboldt. In: Rudolf Hohberg (Hrsg.), Sprache und Bildung. Beiträge zum 150. Todestag Wilhelm von Humboldts. Darmstadt: Techn. Hochschule, 141-173.

Conrad, Ruth (2012): Kirchenbild und Predigtziel. Eine problemgeschichtliche Studie zu ekklesiologischen Dimensionen der Homiletik. Tübingen: Mohr.

E.A. (1836): [Rez.] Berlin, b. Duncker u. Humblot: Ueber die deutschen Universitäten. Ein Gespräch von Dr. Franz Theremin. 1836. 40 S. gr. 8. In: Jenaische Allgemeine LiteraturZeitung 32.2, No. 65, April 1836, $37 \mathrm{f}$.

Eybl, Franz (1998): Jesuitenrhetorik. In: Historisches Wörterbuch der Rhetorik. Hrsg. von Gert Ueding. Bd. 4, 717-728.

Falkmann, Christian Friedrich (1822): Hülfsbuch der deutschen Stylübungen. Hannover: Hahn.

Falkmann, Christian Friedrich (1823): Methodik der deutschen Stylübungen. 2. Aufl. Hannover: Hahn.

Falkmann, Christian Friedrich (1831): Praktische Rhetorik. Hannover: Hahn.

Fichte, Johann Gottlieb (1817): Deduzierter Plan einer höheren Lehranstalt [1807]. Stuttgart: Cotta.

Fichte, Johann Gottlieb (1919): Abschiedsrede in Schul-Pforta am 5. Oktober 1780. In: Neue Fichte-Funde aus der Heimat und Schweiz. Hrsg. von Maximilian Runze. Gotha: Perthes, 31-79.

Fichte, Johann Gottlieb (1970): Gesamtausgabe. Teil III, Bd. 2: Briefe 1793-1795. Hrsg. von Hans Jacob \& Reinhard Lauth. Stuttgart-Bad Cannstatt: Frommann-Holzboog.

Fohrmann, Jürgen (Hrsg.) (2004): Rhetorik. Figuration und Performanz. DFG-Symposion 2002, Stuttgart: J.B. Metzler.

Garve, Christian (1796): Von der Popularität des Vortrags. Breslau: Korn.

Grimm, Jacob \& Wilhelm Grimm (1854): Deutsches Wörterbuch. Leipzig: Hirzel.

Hambsch, Björn (2007): „... ganz andre Beredsamkeit“. Transformationen antiker und moderner Rhetorik bei Johann Gottfried Herder. Tübingen: Niemeyer.

Hambsch, Björn (2011): Verfall der Beredsamkeit. In: Historisches Wörterbuch der Rhetorik. Hrsg. von Gert Ueding. Bd. 10, 1377-1393.

Heinsius, Theodor (1810): Der Redner und Dichter oder Anleitung zur Rede- und Dichtkunst. Berlin: Braunes.

Heinsius, Theodor (1817): Sprach- und Sittenanzeiger der Deutschen. Berlin: Maurer.

Hirt, Aloys (Hrsg.) (1805): Bilderbuch für Mythologie, Archäologie und Kunst. Berlin: Sander. Hösle, Vittorio (2011): Der philosophische Dialog: . MünchenC.H. Beck.

Humboldt, Wilhelm von (1851): Ideen zu einem Versuch, die Grenzen der Wirksamkeit des Staats zu bestimmen. Breslau: Verlag von Eduard Trewendt.

Humboldt, Wilhelm von (1903): Gesammelte Schriften. Hrsg. von Albert Leitzmann. Abt. 1: Werke. Bd. 1: 1785-1795. Berlin: Behr.

Humboldt, Wilhelm von (1907): Ueber den Dualis: gelesen in der Akademie der Wissenschaften am 26. April 1827. In: Gesammelte Schriften. Hrsg. von Albert Leitzmann. Abt. 1: Werke. Band 6, 1. Hälfte: 1827-1835. Berlin: Behr, 4-30.

Humboldt, Wilhelm von (1968): Theorie der Bildung des Menschen. Bruchstück. In: Gesammelte Schriften. Hrsg. von Albert Leitzmann. Abt. 1: Werke. Bd. 1: 1785-1795. Unveränd. photomechan. Nachdr. d. 1. Aufl. Berlin, Behr, 1903. Berlin: De Gruyter, 282-287. 
Humboldt, Wilhelm von (1980): Plan zu einer vergleichenden Anthropologie. In: Werke in fünf Bänden. Hrsg. von Andreas Flitner \& Klaus Giel. Bd. 1: Schriften zur Anthropologie und Geschichte. Darmstadt: WBG, 234-240.

Humboldt, Wilhelm von (1994): Über das vergleichende Sprachstudium in Beziehung auf die verschiedenen Epochen der Sprachentwicklung. In: Über die Sprache. Reden vor der Akademie. Hrsg. von Jürgen Trabant. Tübingen, Basel: Francke, 11-32.

Humboldt, Wilhelm von (2010): Über die innere und äussere Organisation der höheren wissenschaftlichen Anstalten in Berlin. In: Werke in fünf Bänden. Bd. 4: Schriften zur Politik und zum Bildungswesen. Hrsg. von Andreas Flitner \& Klaus Giel. Darmstadt: WBG, 255-265.

Kant, Immanuel (1908): Kritik der Urtheilskraft. In: Kant's Gesammelte Schriften. Bd. V, 1. Hrsg. von der Preußischen Akademie der Wissenschaften. Berlin: Reimer.

Keller, Andreas (2008): Frühe Neuzeit. Das rhetorische Zeitalter. Berlin: Akademie.

Kost, Jürgen (2004): Wilhelm von Humboldt, Weimarer Klassik, Bürgerliches Bewußtsein. Kulturelle Entwürfe in Deutschland um 1800. Würzburg: Königshausen \& Neumann.

Krause, Peter D. (2001): Unbestimmte Rhetorik. Friedrich Schlegel und die Redekunst um 1800. Tübingen: Niemeyer.

Lindner, Johann Gotthilf (1755): Anweisung zur guten Schreibart überhaupt und zur Beredsamkeit insonderheit. Königsberg: Hartung.

Lingelbach, Gabriele (Hrsg.) (2006): Vorlesung, Seminar, Repetitorium. Universitäre geschichtswissenschaftliche Lehre im historischen Vergleich. München: Meidenbauer.

Lohmann, Ingrid (1993): Bildung, bürgerliche Öffentlichkeit und Beredsamkeit: zur pädagogischen Transformation der Rhetorik zwischen 1750 und 1850. Münster: De Gruyter.

Lorenz, Kuno (1973): Der dialogische Wahrheitsbegriff. In: Neue Hefte für Philosophie 2/3, $11-123$.

Martyn, David (2014): Rhetorik der Muttersprache. In: Ralf Simon (Hrsg.), Herders Rhetoriken im Kontext des 18. Jahrhunderts. Beiträge zur Konferenz der Internationalen HerderGesellschaft Schloss Beuggen nahe Basel 2012. Heidelberg: Synchron, 49-64.

Melos, J.G. (Hrsg.) (1818): Mustersammlung zu Declamationsübungen für die Jugend. Leipzig: Hartmann.

Müller, Adam (1967): Zwölf Reden über die Beredsamkeit und deren Verfall in Deutschland. Mit einem Essay und einem Nachw. von Walter Jens. Frankfurt a.M.: Insel.

Müller, Ernst (2016): Masken der Aufklärung. Theologische Rhetorik und Säkularisierung. In: Weimarer Beiträge 62, 5-36.

o.A. (1780): Die deutsche Beredsamkeit in der Ausübung oder: Sammlung deutscher Muster zur Bildung eines deutschen Redners. Frankfurt a.M., Leipzig: Haug.

o.A. [i. e. Fichte] (1788): Versuch über die Beredsamkeit, nur für meine Zuhörer bestimmt. In: Kritische Uebersicht der neuesten schönen Litteratur der Deutschen. Leipzig: Göschen, 31-39.

Oesterreich, Peter L. (1997): Das gelehrte Absolute. Metaphysik und Rhetorik bei Kant, Fichte und Schelling. Darmstadt: WBG.

Paulsen, Friedrich (1912): Gesammelte pädagogische Abhandlungen. Hrsg. von Eduard Spranger. Stuttgart, Berlin: Cotta.

Paracelsus (1590): Der Bücher und Schrifften des [. . .] Philippi Theophrasti Bombast von Hohenheim Paracelsi genannt. 9. Teil. Basel: Waldkirch. 
Petri, Friedrich Erdmann (1831): Rhetorisches Wörterbüchlein, zunächst für Gelehrten-Schulen. Leipzig: Arnoldische Buchh.

Purgold, Ludwig (1807): Über die Bildung zur Poesie und Beredsamkeit auf Schulen. Nebst Probearbeiten der Gymnasiasten zu Wiburg. St. Petersburg: Drechsler.

Ptassek, Peter (1993): Rhetorische Rationalität. Stationen einer Verdrängungsgeschichte von der Antike bis zur Neuzeit: . München Wilhelm Fink.

Ritter, Carl (1852): Einleitung zu dem Versuche einer allgemeinen vergleichenden Geographie. In dem Jahre 1818 geschrieben. Berlin: Reimer.

Rosen, Valeska von (2012): Erosionen der Rhetorik? Strategien der Ambiguität in den bildenden Künsten, Dichtung und Musik. Einleitende Überlegungen. In: Dies. (Hrsg.), Erosionen der Rhetorik? Strategien der Ambiguität in den Künsten der Frühen Neuzeit. Wiesbaden: Culturae 4, 1-28.

Schierbaum, Martin (2002): Friedrich von Hardenbergs poetisierte Rhetorik. Politische Ästhetik der Frühromantik. Paderborn: Schöningh.

Schiewe, Jürgen (1996): Sprachenwechsel, Funktionswandel, Austausch der Denkstile. Die Universität Freiburg zwischen Latein und Deutsch. Tübingen: Niemeyer.

Schleiermacher, Friedrich Daniel Ernst (1988): Dialektik (1814/15). Einleitung zur Dialektik. (1833). Hrsg. von Andreas Arndt. Hamburg: Meiner.

Schmid, Susanne (2006): Gespräch, Geselligkeit und Einsamkeit um 1800. In: GermanischRomanische Monatsschrift 56, 45-58.

Schnyder, Peter (1999): Die Magie der Rhetorik. Poesie, Philosophie und Politik in Friedrich Schlegels Frühwerk. Paderborn: Schöningh.

Spenkuch, Hartwin (2010): Die Politik des Kultusministeriums gegenüber den Wissenschaften und den Hochschulen. In: Berlin-Brandenburgischen Akademie der Wissenschaften unter Leitung von Wolfgang Neugebauer (Hrsg.), Das Kultusministerium auf seinen Wirkungsfeldern Schule, Wissenschaft, Kirchen, Künste und Medizinalwesen. Berlin: Akademie, 157-161.

Tenorth, Heinz-Elmar (Hrsg.) (2012a): Geschichte der Universität Unter den Linden. Bd. 1: Gründung und Blütezeit der Universität zu Berlin 1810-1918. Berlin: Akademie, XV-XLIII.

Tenorth, Heinz-Elmar (2012b): Geschichte der Universität zu Berlin, 1810 bis 2010. Zur Einleitung. In: Ders. (Hrsg.), Geschichte der Universität Unter den Linden. Bd. 1: Gründung und Blütezeit der Universität zu Berlin 1810-1918. Berlin: Akademie, XV-XLIII.

Tenorth, Heinz-Elmar (2018): Wilhelm von Humboldt: Bildungspolitik und Universitätsreform. Paderborn: Schöningh.

Theremin, Franz (1836): Über die deutschen Universitäten. Ein Gespräch. Berlin: Duncker. Theremin, Franz (1837): Die Beredsamkeit eine Tugend, oder Grundlinien einer systematischen Rhetorik. Berlin: Duncker.

Till, Dietmar (2004a): Anthropologie oder System? Ein Plädoyer für Entscheidungen. In: Rhetorik. Internationales Jahrbuch 23, 11-25.

Till, Dietmar (2004b): Transformationen der Rhetorik. Untersuchungen zum Wandel der Rhetoriktheorie im 17. und 18. Jahrhundert. Tübingen: Niemeyer.

Treß, Werner (2012): Professoren - Der Lehrkörper und seine Praxis zwischen Wissenschaft, Politik und Gesellschaft. In: Heinz-Elmar Tenorth (Hrsg.), Geschichte der Universität Unter den Linden. Bd. 1: Gründung und Blütezeit der Universität zu Berlin 1810-1918. Berlin: Akademie, 131-207.

Tschong, Youngkun (1991): Charakter und Bildung. Zur Grundlegung von Wilhelm von Humboldts bildungstheoretischem Denken. Würzburg: Königshausen \& Neumann. 
Viermond, Wolfgang (2011) (Hrsg.): Die Vorlesungen der Berliner Universität. 1810-1834.

Berlin: Akademie.

Voßkamp, Wilhelm (2004): „Ein anderes Selbst“. Bild und Bildung im deutschen Roman des 18. und 19. Jahrhunderts. Göttingen: Wallstein.

Wackernagel, Wilhelm (2003): Poetik, Rhetorik und Stilistik. Academische Vorlesungen. Hrsg. von Ludwig Sieber, Halle 1873. Nachdr. Hildesheim: Olms.

Weber, Philipp (2017): Kosmos und Subjektivität in der Frühromantik. München: Wilhelm Fink. Weigel, Valentin (1695): Studium universale [. . .]. [Druck posthum.] Frankfurt, Leipzig: Müller. Wriedt, Markus (2017): Bildung, Schule und Universität. In: Günther Frank (Hrsg.), Philipp Melanchthon: Der Reformator zwischen Glauben und Wissen. Ein Handbuch. Berlin, Boston: De Gruyter.

Zöllner, Detlef (1989): Wilhelm von Humboldt. Einbildung und Wirklichkeit. Das bildungstheoretische Fundament seiner Sprachphilosophie. Münster, New York: Waxmann. 